\title{
Hot HB stars in globular clusters - Physical parameters and consequences for theory
}

\section{The second parameter pair M 3 and $M 13^{\star}$}

\author{
S. Moehler ${ }^{1,2}$, W. B. Landsman ${ }^{3}$, A. V. Sweigart ${ }^{4}$, and F. Grundahl ${ }^{5}$ \\ 1 Institut für Theoretische Physik und Astrophysik, Abteilung Astrophysik, Leibnizstraße 15, 24098 Kiel, Germany \\ 2 Dr. Remeis-Sternwarte, Astronomisches Institut der Universität Erlangen-Nürnberg, Sternwartstr. 7, 96049 Bamberg, \\ Germany \\ 3 SSAI, NASA Goddard Space Flight Center, Code 681, Greenbelt, MD 20771, USA \\ e-mail: landsman@mpb.gsfc.nasa.gov \\ 4 NASA Goddard Space Flight Center, Code 681, Greenbelt, MD 20771, USA \\ e-mail: sweigart@bach.gsfc.nasa.gov \\ 5 Institute of Physics and Astronomy, Aarhus University, Ny Munkegade, 8000 Aarhus C, Denmark \\ e-mail: fgj@ifa.au.dk
}

Received 4 October 2002 / Accepted 23 April 2003

\begin{abstract}
We present the results of spectroscopic analyses of hot horizontal branch (HB) stars in M 13 and M 3, which form a famous "second parameter" pair. From the spectra and Strömgren photometry we derived - for the first time in M 13 atmospheric parameters (effective temperature and surface gravity). For stars with Strömgren temperatures between 10000 and $12000 \mathrm{~K}$ we found excellent agreement between the atmospheric parameters derived from Strömgren photometry and those derived from Balmer line profile fits. However, for cooler stars there is a disagreement in the parameters derived by the two methods, for which we have no satisfactory explanation. Stars hotter than $12000 \mathrm{~K}$ show evidence for helium depletion and iron enrichment, both in M 3 and M 13. Accounting for the iron enrichment substantially improves the agreement with canonical evolutionary models, although the derived gravities and masses are still somewhat too low. This remaining discrepancy may be an indication that scaled-solar metal-rich model atmospheres do not adequately represent the highly non-solar abundance ratios found in blue HB stars affected by diffusion. We discuss the effects of an enhancement in the envelope helium abundance on the atmospheric parameters of the blue HB stars, as might be caused by deep mixing on the red giant branch or primordial pollution from an earlier generation of intermediate mass asymptotic giant branch stars.
\end{abstract}

Key words. stars: atmospheres - stars: evolution - stars: horizontal-branch - Galaxy: globular clusters: individual: M 3 Galaxy: globular clusters: individual: M 13

\section{Introduction}

The two globular clusters M 3 and M 13 form a famous "second parameter" pair of clusters that show very different horizontal branch $(\mathrm{HB})$ morphologies despite quite similar metallicities $([\mathrm{Fe} / \mathrm{H}]=-1.57$ for $\mathrm{M} 3$ and -1.54 for $\mathrm{M} 13$, Harris 1996): M 3 possesses a horizontal HB populated from the red to the blue end, while M 13 exhibits a predominantly blue HB followed by a very long blue tail that extends to

Send offprint requests to: $\mathrm{S}$. Moehler, e-mail: moehler@astrophysik.uni-kiel.de

* Based on observations collected at the German-Spanish Astronomical Center (DSAZ), Calar Alto, operated by the MaxPlanck-Institut für Astronomie Heidelberg jointly with the Spanish National Commission for Astronomy. temperatures of $\approx 35000 \mathrm{~K}$ (Parise et al. 1998). No consensus has yet been reached on the reasons for this difference in HB morphology. While it has often been argued that age, one of the most widely discussed 2nd parameter candidates (Sarajedini et al. 1997), cannot be the 2nd parameter for this pair (Catelan $\&$ de Freitas Pacheco 1995; Ferraro et al. 1997; Paltrinieri et al. 1998; Grundahl 1999), the subject remains controversial (e.g. Rey et al. 2001). VandenBerg (2000), in particular, has emphasized that something other than age must be different between M 3 and M 13.

M 13 (along with $\omega$ Cen) harbors the most dramatic examples of stars presenting abundance anomalies on the upper red giant branch (RGB; Kraft et al. 1993, 1995, 1997; Cavallo \& Nagar 2000), whereas the abundance anomalies in M 3 are considerably less pronounced (Kraft et al. 1992; 
Cavallo \& Nagar 2000). In particular, the super-oxygen poor stars found near the tip of the RGB in M 13 are absent in M 3. These abundance anomalies may arise from deep mixing processes which bring up nuclearly processed material from the vicinity of the hydrogen burning shell during a star's RGB phase. Support for this possibility comes from the larger $\mathrm{Na}$ and $\mathrm{Al}$ abundances and smaller $\mathrm{O}$ and $\mathrm{Mg}$ abundances found in stars near the tip of the RGB in M 13 compared to stars further down the RGB (Kraft 1999; Cavallo \& Nagar 2000). Alternatively, the recent detections of similar abundance anomalies among main-sequence stars in a few clusters (Cannon et al. 1998; Gratton et al. 2001) suggest a primordial origin, perhaps due to pollution from an earlier generation of intermediate mass asymptotic giant branch (AGB) stars.

Both the deep mixing and primordial scenarios for the origin of the abundance anomalies may have consequences for the luminosity and morphology of the HB, as outlined by Sweigart (1997b) and D'Antona et al. (2002), respectively. Briefly, in the deep-mixing scenario, helium from the H-burning shell is mixed into the envelope on the RGB and thereby causes a star to have a bluer and more luminous position on the HB. In the primordial scenario, stars on the main-sequence which have been polluted by the products of an earlier AGB generation have a higher helium abundance. These polluted stars will then have a lower turnoff mass for a given age than the unpolluted cluster stars with a normal helium abundance, and thus will be more likely to end up on the blue end of the HB. As in the deep-mixing scenario, the primordial scenario also predicts a higher luminosity of the hydrogen shell on the $\mathrm{HB}$, due to the increased helium abundance.

Thus, both the deep mixing and primordial scenarios predict that the stars with the strongest abundance anomalies should end up on the bluest part of the HB, and have both a higher helium abundance and higher luminosity (and lower gravity) than the HB stars with normal abundances. Unfortunately, a straightforward test of these predictions is complicated by the processes of diffusion and radiative levitation in HB stars. The observed photospheric helium abundance in hot HB stars, for example, has long been known to be strongly depleted (see Moehler 2001 for an overview) presumably due to gravitational settling. Moreover, the use of luminosity or gravity discriminants for testing these scenarios is complicated by the supersolar iron abundances in HB stars hotter than $\approx 11500 \mathrm{~K}$ (Glaspey et al. 1989; Behr et al. 1999, 2000) due to radiative levitation (Michaud et al. 1983). If the hot HB stars with enhanced abundances are analysed with model atmospheres at the cluster abundance, the stars appear to have anomalously low gravities (Moehler et al. 2000) or to be anomalously bright in certain bandpasses, such as Strömgren $u$ (Grundahl et al. 1999). However, when Moehler et al. (2000) used model atmospheres with the correct iron abundance for the gravity determinations of hot HB stars in NGC 6752, they found that the "low-gravity" anomaly mostly disappeared, although a small discrepancy remained for stars with $15000 \mathrm{~K}<T_{\text {eff }}<20000 \mathrm{~K}$. Interestingly, Parise et al. (1998) also detect a possible luminosity offset of M 13 HB stars in this temperature range from their $1620 \AA$ photometry obtained with the Ultraviolet Imaging Telescope (UIT).
Although selected hot HB stars in M 13 have been observed for an abundance analysis (Behr et al. 1999) and studied in Strömgren photometry (Grundahl et al. 1999), there have been no previous spectroscopic studies to obtain temperatures and gravities. Here we present spectroscopy of 22 hot HB candidates in M 13 to derive effective temperatures and surface gravities in order to search for any deviations from canonical HB models. We also estimate abundances of helium, magnesium, and iron. Observations of four hot HB stars in M 3 serve as a control sample.

\section{Observations}

We selected our targets in M 13 from the Strömgren photometry of Grundahl et al. (1998, see Table 1) and those in M 3 from the Johnson photometry of Buonanno et al. (1994, see Table 2). We also included three targets in M 13 from Behr et al. (1999) for comparison. Our targets are plotted along the HBs of M 13 and M 3 in Fig. 1. For our observations we used the Calar Alto $3.5 \mathrm{~m}$ telescope with the TWIN spectrograph. While we observed in both channels (blue and red) the crowding and consequent stray light of the primarily red cluster stars made the data from the red channel very difficult to reduce and analyse. We will therefore limit ourselves to the discussion of the data from the blue channel. There we used CCD\#11 $\left(\right.$ SITe\#12a, $800 \times 2000$ pixels, $(15 \mu \mathrm{m})^{2}$ pixel size, read-out noise $6 \mathrm{e}^{-}$, conversion factor $1.1 \mathrm{e}^{-} /$count) and grating $\mathrm{T} 12$ (72 $\AA \mathrm{mm}^{-1}$ ) to cover a wavelength range of $3410 \AA-5570 \AA$. Combined with a slit width of $1.5^{\prime \prime}$ we thus achieved a mean spectral resolution of $3.4 \AA$ as determined from the FWHM of the wavelength calibration lines. The spectra were obtained on June 4-6, 1999. For calibration purposes we observed each night ten bias frames and ten dome flat-fields with a mean exposure level of about 10000 counts each. Before and after each science observation we took HeAr spectra for wavelength calibration purposes. We observed dark frames of 3600 and $1800 \mathrm{~s}$ duration to measure the dark current of the CCD. As flux standard stars we used Feige 56, HZ 44, BD+28 4211 , and HZ 21.

In order to observe as many stars as possible we oriented the slit to cover up to four hot HB stars at once. This of course did not allow us to reduce the light loss due to atmospheric dispersion by observing along the parallactic angle and also required observations in fairly crowded regions.

\section{Data reduction}

We first averaged the bias and flat field frames separately for each night. The mean bias frames of the second and the third night showed the same level of about 923 counts (and were therefore averaged), whereas the one from the first night had on average 877 counts. The same difference was found in the overscan regions of the respective bias frames. We therefore determined the mean overscan of each science frame before deciding which bias frame to use (which was then adjusted to the individual overscan level of the science frame). The mean dark current determined from long dark frames showed no structure and turned out to be negligible $(1.5 \pm 2$ counts/hr/pixel $)$. 
Table 1. Coordinates, photometric data, and heliocentric radial velocities for target stars in M 13 (Grundahl et al. 1998 [G]; Piotto et al. 2002 [WF3035, WF3485]; this paper [WF3085, WFPC2 data reduced as described by Dolphin 2000]). H $\beta$ indices with a large ( $>0$.05) uncertainty are marked with a colon.

\begin{tabular}{|c|c|c|c|c|c|c|c|}
\hline Star & $\alpha_{2000}$ & $\delta_{2000}$ & $y$ & $b-y$ & $u-b$ & $\beta$ & $\begin{array}{l}v_{\mathrm{rad}} \\
{\left[\mathrm{km} \mathrm{s}^{-1}\right]}\end{array}$ \\
\hline G43 & $16^{\mathrm{h}} 41^{\mathrm{m}} 34.7$ & $+36^{\circ} 29^{\prime} 13^{\prime \prime} .7$ & 14.262 & -0.021 & +0.688 & 2.651 & -287 \\
\hline G129 & $16^{\mathrm{h}} 41^{\mathrm{m}} 41^{\mathrm{s}} .8$ & $+36^{\circ} 29^{\prime} 20^{\prime} 5$ & 14.918 & $+0^{\mathrm{m}} \cdot 053$ & $+1 \mathrm{~m} \cdot 632$ & 2.825 & -257 \\
\hline G137 & $16^{\mathrm{h}} 41^{\mathrm{m}} 46^{\mathrm{s}} .3$ & $+36^{\circ} 30^{\prime} 12^{\prime \prime} .6$ & $15^{\mathrm{m}} \cdot 019$ & $+0^{\mathrm{m}} .040$ & $+1^{\mathrm{m}} \cdot 584$ & 2.837 & -251 \\
\hline G195 & $16^{\mathrm{h}} 41^{\mathrm{m}} 43^{\mathrm{s}} .1$ & $+36^{\circ} 30^{\prime} 08^{\prime \prime} .1$ & $15^{\mathrm{m}} \cdot 159$ & $+0^{\mathrm{m}} 040$ & $+1^{\mathrm{m}} \cdot 554$ & 2.836 & -229 \\
\hline G201 & $16^{\mathrm{h}} 41^{\mathrm{m}} 47^{\mathrm{s}} .9$ & $+36^{\circ} 29^{\prime} 47^{\prime \prime} .3$ & $15^{\mathrm{m}} \cdot 237$ & $+0^{\mathrm{m}} 025$ & $+1^{\mathrm{m}} .485$ & 2.832 & -276 \\
\hline G208 & $16^{\mathrm{h}} 41^{\mathrm{m}} 34^{\mathrm{s}} .1$ & $+36^{\circ} 29^{\prime} 51^{\prime \prime} .4$ & $15^{\mathrm{m}} \cdot 298$ & $+0^{\mathrm{m}} 016$ & $+1^{\mathrm{m}} \cdot 347$ & 2. $820:$ & -244 \\
\hline G219 & $16^{\mathrm{h}} 41^{\mathrm{m}} 39^{\mathrm{s}} .1$ & $+36^{\circ} 29^{\prime} 54^{\prime \prime} .0$ & $15^{\mathrm{m}} \cdot 222$ & $+0^{\mathrm{m}} 039$ & $+1^{\mathrm{m}} \cdot 564$ & 2.844 & -263 \\
\hline G235 & $16^{\mathrm{h}} 41^{\mathrm{m}} 40 \mathrm{~s} .1$ & $+36^{\circ} 29^{\prime} 00^{\prime} 1$ & $15^{\mathrm{m}} \cdot 245$ & $+0^{\mathrm{m}} 033$ & $+1^{\mathrm{m}} \cdot 528$ & 2.843 & -245 \\
\hline G297 & $16^{\mathrm{h}} 41^{\mathrm{m}} 35^{\mathrm{s}} .9$ & $+36^{\circ} 29^{\prime} 27^{\prime \prime} .0$ & $15^{\mathrm{m}} \cdot 626$ & $+0^{\mathrm{m}} \cdot 002$ & $+1^{\mathrm{m}} \cdot 210$ & 2.795 & -245 \\
\hline G314 & $16^{\mathrm{h}} 41^{\mathrm{m}} 39^{\mathrm{s}} .9$ & $+36^{\circ} 30^{\prime} 00^{\prime} .5$ & $15^{\mathrm{m}} \cdot 638$ & $+0{ }^{\mathrm{m}} 004$ & $+1^{\mathrm{m}} \cdot 207$ & 2.804 & -268 \\
\hline G322 & $16^{\mathrm{h}} 41^{\mathrm{m}} 40.7$ & $+36^{\circ} 28^{\prime} 52^{\prime \prime} .5$ & $15^{\mathrm{m}} \cdot 865$ & $-0^{\mathrm{m}} 018$ & $+0^{\mathrm{m}} 711$ & $2 \mathrm{~m} 696$ & -246 \\
\hline G342 & $16^{\mathrm{h}} 41^{\mathrm{m}} 38^{\mathrm{s}} .8$ & $+36^{\circ} 30^{\prime} 42^{\prime \prime} .4$ & $15^{\mathrm{m}} \cdot 840$ & $-0^{\mathrm{m}} \cdot 002$ & $+1^{\mathrm{m}} .036$ & 2.766 & -231 \\
\hline G382 & $16^{\mathrm{h}} 41^{\mathrm{m}} 41^{\mathrm{s}} .1$ & $+36^{\circ} 29^{\prime} 39^{\prime} ._{1}$ & $16^{\mathrm{m}} 058$ & $-0^{\mathrm{m}} 021$ & $+0^{\mathrm{m}} 649$ & 2.674 & -255 \\
\hline G496 & $16^{\mathrm{h}} 41^{\mathrm{m}} 41^{\mathrm{s}} .1$ & $+36^{\circ} 30^{\prime} 05^{\prime \prime} .7$ & 16.538 & $-0{ }^{\mathrm{m}} 036$ & +0.384 & $2 \mathrm{~m} 673$ & -231 \\
\hline G503 & $16^{\mathrm{h}} 41^{\mathrm{m}} 33^{\mathrm{s}} .8$ & $+36^{\circ} 30^{\prime} 15^{\prime \prime} .5$ & $16^{\mathrm{m}} .531$ & -0.035 & +0.532 & 2. $713:$ & -221 \\
\hline G635 & $16^{\mathrm{h}} 41^{\mathrm{m}} 40{ }^{\mathrm{s}} .5$ & $+36^{\circ} 31^{\prime} 19^{\prime} 7$ & $16^{\mathrm{m}} \cdot 957$ & -0.041 & $+0^{\mathrm{m}} 316$ & $2^{\mathrm{m}} \cdot 696:$ & -246 \\
\hline G744 & $16^{\mathrm{h}} 41^{\mathrm{m}} 48^{\mathrm{s}} .5$ & $+36^{\circ} 29^{\prime} 59^{\prime} 4$ & $17 \cdot 229$ & -0.054 & +0.266 & $2^{\mathrm{m}} \cdot 679:$ & -251 \\
\hline G781 & $16^{\mathrm{h}} 41^{\mathrm{m}} 48^{\mathrm{s}} .8$ & $+36^{\circ} 29^{\prime} 28^{\prime \prime} .2$ & $17 \cdot 348$ & -0.054 & $+0^{\mathrm{m}} \cdot 240$ & 2. $661:$ & -237 \\
\hline G827 & $16^{\mathrm{h}} 41^{\mathrm{m}} 41^{\mathrm{s}} .5$ & $+36^{\circ} 30^{\prime} 37^{\prime \prime} .0$ & 17.530 & $-0{ }^{\mathrm{m}} 072$ & $+0^{\mathrm{m}} \cdot 168$ & $2 \mathrm{~m} \cdot 671$ & -252 \\
\hline Star & $\alpha_{2000}$ & $\delta_{2000}$ & $V$ & $B-V$ & & & $\begin{array}{l}v_{\mathrm{rad}} \\
{\left[\mathrm{km} \mathrm{s}^{-1}\right]}\end{array}$ \\
\hline WF 3035 & $16^{\mathrm{h}} 41^{\mathrm{m}} 48^{\mathrm{s}} .1$ & $+36^{\circ} 28^{\prime} 20^{\prime} 6$ & 15.34 & $-0 \mathrm{~m} 01$ & & & -288 \\
\hline WF 3085 & $16^{\mathrm{h}} 41^{\mathrm{m}} 37^{\mathrm{s}} .8$ & $+36^{\circ} 26^{\prime} 37^{\prime \prime} .1$ & $16^{\mathrm{m}} \cdot 15$ & -0.10 & & & -249 \\
\hline WF 3485 & $16^{\mathrm{h}} 41^{\mathrm{m}} 36^{\mathrm{s}} .9$ & $+36^{\circ} 26^{\prime} 47^{\prime \prime} .7$ & $16^{\mathrm{m}} \cdot 25$ & -0.06 & & & -243 \\
\hline
\end{tabular}

We determined the spectral energy distribution of the flat field lamp by averaging the mean flat fields of each night along the spatial axis. These one-dimensional "flat field spectra" were then heavily smoothed and used afterwards to normalize the dome flats along the dispersion axis. As the normalized flat fields of each night differed slightly from each other we always corrected the science spectra using the flat field obtained for the same night.

For the wavelength calibration we fitted 2nd-order polynomials to the dispersion relations of the HeAr spectra (using 46 unblended lines) which resulted in mean residuals of $\leq 0.15 \AA$. We rebinned the frames two-dimensionally to constant wavelength steps. Before the sky fit the frames were smoothed along the spatial axis to erase cosmic ray hits in the background. To determine the sky background we had to find regions without any stellar spectra, which were sometimes not close to the place of the object's spectrum. Nevertheless the flat field correction and wavelength calibration turned out to be good enough that a linear fit to the spatial distribution of the sky light allowed the sky background at the object's position to be reproduced with sufficient accuracy. This means in our case that after the fitted sky background was subtracted from the
Table 2. Coordinates, photometric data, and heliocentric radial velocities for the target stars in M 3 (Buonanno et al. 1994).

\begin{tabular}{llllll}
\hline \hline Star & $\alpha_{2000}$ & $\delta_{2000}$ & $V$ & $B-V$ & $\begin{array}{l}v_{\text {rad }} \\
{\left[\mathrm{km} \mathrm{s}^{-1}\right]}\end{array}$ \\
\hline B254 & $13^{\mathrm{h}} 42^{\mathrm{m}} 05^{\mathrm{s}} \cdot 3$ & $+28^{\circ} 25^{\prime} 09^{\prime}$ & $16^{\mathrm{m}} \cdot 14$ & $-0^{\mathrm{m}} \cdot 03$ & -151 \\
B352 & $13^{\mathrm{h}} 42^{\mathrm{m}} 28^{\mathrm{s}} .8$ & $+28^{\circ} 24^{\prime} 33^{\prime \prime}$ & $17^{\mathrm{m}} \cdot 01$ & $-0^{\mathrm{m}} \cdot 23$ & -149 \\
B445 & $13^{\mathrm{h}} 42^{\mathrm{m}} 33.4$ & $+28^{\circ} 23^{\prime} 57^{\prime \prime}$ & $16^{\mathrm{m}} \cdot 27$ & $-0^{\mathrm{m}} \cdot 06$ & -146 \\
B621 & $13^{\mathrm{h}} 41^{\mathrm{m}} 56^{\mathrm{s}} \cdot 0$ & $+28^{\circ} 22^{\prime} 54^{\prime \prime}$ & $16^{\mathrm{m}} \cdot 55$ & $-0^{\mathrm{m}} \cdot 10$ & -177 \\
\hline
\end{tabular}

unsmoothed frame we do not see any absorption lines caused by the predominantly red stars of the clusters. The skysubtracted spectra were extracted using Horne's (1986) algorithm as implemented in MIDAS (Munich Image Data Analysis System).

Finally the spectra were corrected for atmospheric extinction using the extinction coefficients for La Silla (Tüg 1977) as implemented in MIDAS. The data for the flux standard stars were taken from Hamuy et al. (1992, Feige 56) and Oke (1990, all others) and the response curves were fitted by splines or high-order polynomials. The flux-calibration is helpful for the 


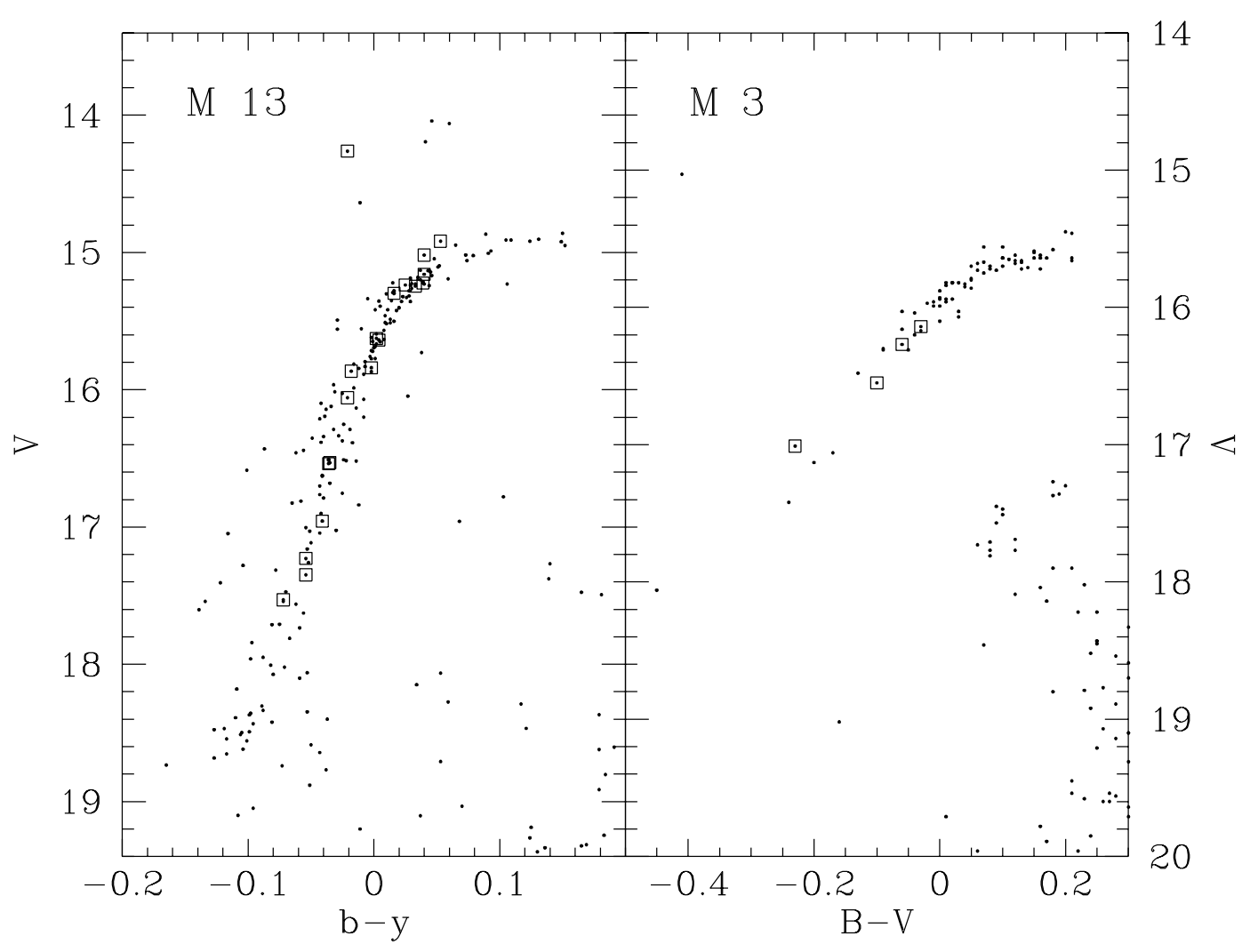

Fig. 1. The horizontal branches of M 13 (Grundahl et al. 1998, left panel) and M 3 (Buonanno et al. 1994, right panel) with the spectroscopic targets marked by open squares.

later normalization of the spectra as it takes out all large-scale sensitivity variations of the instrumental setup. Atmospheric dispersion will cause light loss especially at blue end of the spectral range so that the flux distribution of the calibrated spectra cannot be used to infer temperatures or gravities (e.g. from the Balmer jump).

We derived radial velocities from the positions of the Balmer and helium lines. The resulting heliocentric velocities are listed in Tables 1 and 2. The error of the velocities (as estimated from the scatter of the velocities derived from individual lines) is about $30 \mathrm{~km} \mathrm{~s}^{-1}$. The average radial velocity of the M 13 stars of $-251 \mathrm{~km} \mathrm{~s}^{-1}$ agrees well with the literature value of $-246 \mathrm{~km} \mathrm{~s}^{-1}$ (Lupton et al. 1987) within the error bars. The same is true for M 3 with $-156 \mathrm{~km} \mathrm{~s}^{-1}$ (our result) vs. $-147 \mathrm{~km} \mathrm{~s}^{-1}$ (Pryor et al. 1988). Individual velocities may deviate considerably from the mean value because the stars may not have been in the center of the slit along the dispersion axis during the observation due to the placement of the slit to include several target stars simultaneously.

The Doppler-corrected spectra were then co-added and normalized by eye and are plotted in Fig. 2 .

\section{Atmospheric parameters}

\subsection{Photometric estimates}

To get a first estimate of effective temperatures and surface gravities we used the Strömgren $u v b y \beta$ photometry given in Table 1.
Because an empirical calibration of the Strömgren indices is not yet available for hot HB stars, we used the Strömgren indices (including $\mathrm{H} \beta$ ) computed with the ATLAS9 code by Castelli (1998) to derive values for $T_{\text {eff }}$ and $\log g$. We adopted the model with $[\mathrm{Fe} / \mathrm{H}]=-1.5$ with $\alpha$ elements enhanced by +0.4 dex, a solar helium abundance and a microturbulent velocity of $1 \mathrm{~km} \mathrm{~s}^{-1}$. The normalization of the $\mathrm{H} \beta$ indices by Castelli uses the values for the Sun and Vega as anchor points for a linear interpolation relating the calculated indices, $\beta_{\text {calc }}$ to the observed indices, $\beta_{\mathrm{obs}}$

$\beta_{\mathrm{obs}}=1.287 \beta_{\mathrm{calc}}+2.5298$.

The observed Strömgren $u v b y$ photometry was dereddened by $E_{B-V}=0$. 02 .

For the stars with $b-y<0$ m $01(\approx 11000 \mathrm{~K})$, the best-fit values of $T_{\text {eff }}$ and $\log g$ were determined by minimizing the $\chi^{2}$ difference between the observed and calculated $u v b y$ and $\mathrm{H} \beta$ photometry. For the stars with $b-y>0$. 01 , we computed theoretical $(a, r)$ indices

$$
\begin{aligned}
& a=1.36 \cdot(b-y)+0.36 \cdot m 0+0.18 \cdot c 0-0.2448 \\
& r=-0.07 \cdot(b-y)+0.35 \cdot c 0-(\beta-2.565)
\end{aligned}
$$

where for stars with $8500 \mathrm{~K}<T_{\text {eff }}<11000 \mathrm{~K} a$ correlates with $T_{\text {eff }}$ and $r$ correlates with $\log g$ (Napiwotzki et al. 1993). The $(a, r)$ method was also used for field HB stars with similar Strömgren colours by Kinman et al. (2000). The values of $T_{\text {eff }}$ and $\log g$ derived from Strömgren photometry are given 


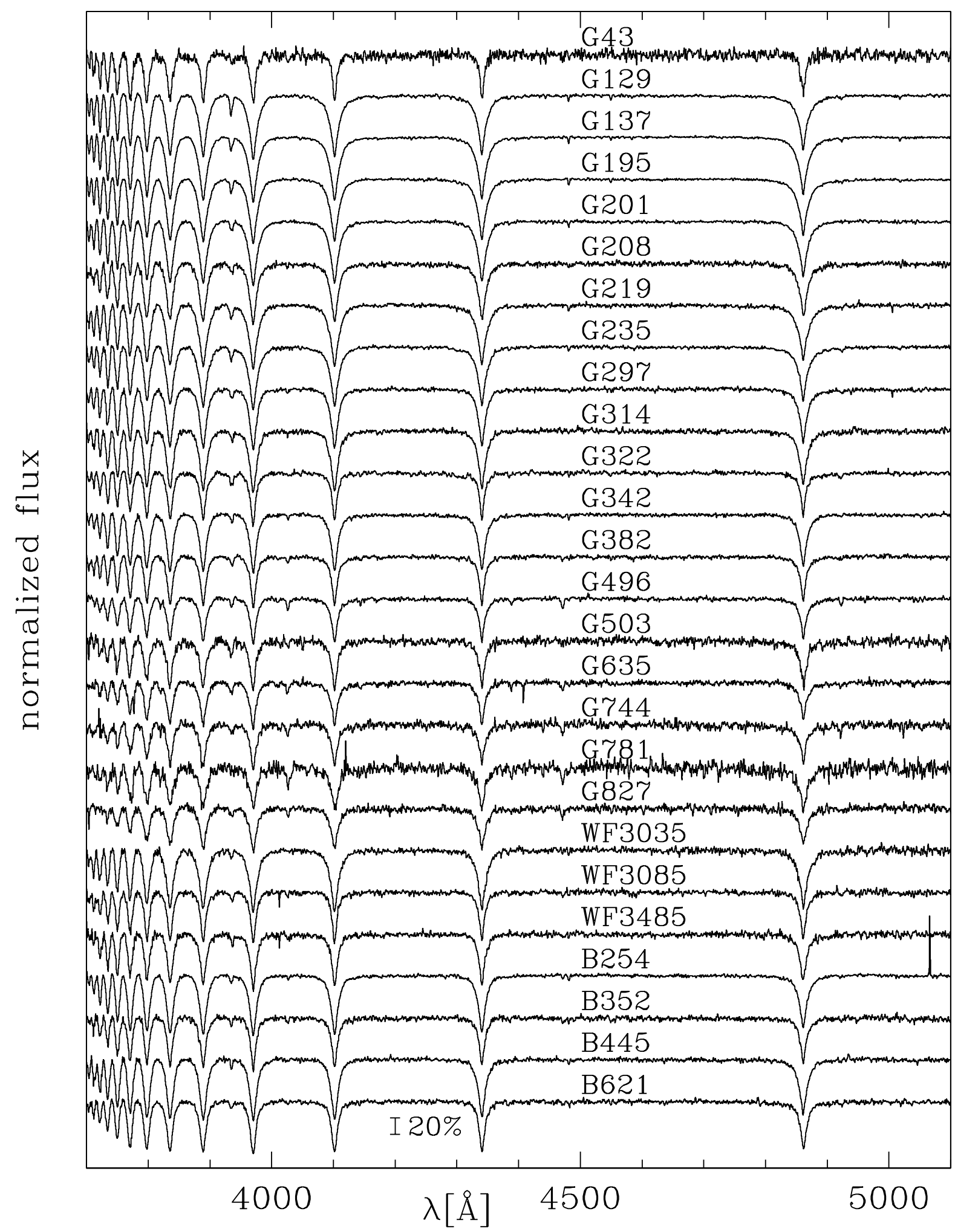

Fig. 2. Normalized spectra of the programme stars in M 13 and M 3. The part shortward of $3900 \AA$ was normalized by taking the highest flux point as continuum value.

in Table 3 (Cols. 2 and 3) and plotted in the upper panel of Fig. 3. These values were then used as starting points for the fits of model spectra to the observed Balmer lines in order to derive effective temperatures and surface gravities (see below). A prior idea of the probable range in temperature and gravity is especially helpful for stars near the Balmer maximum, where the fit of the spectral lines may yield different results depending on the starting values, although the solutions on the two sides of the Balmer maximum usually show quite different values of $\chi^{2}$. For reasons of consistency we used the photometrically 


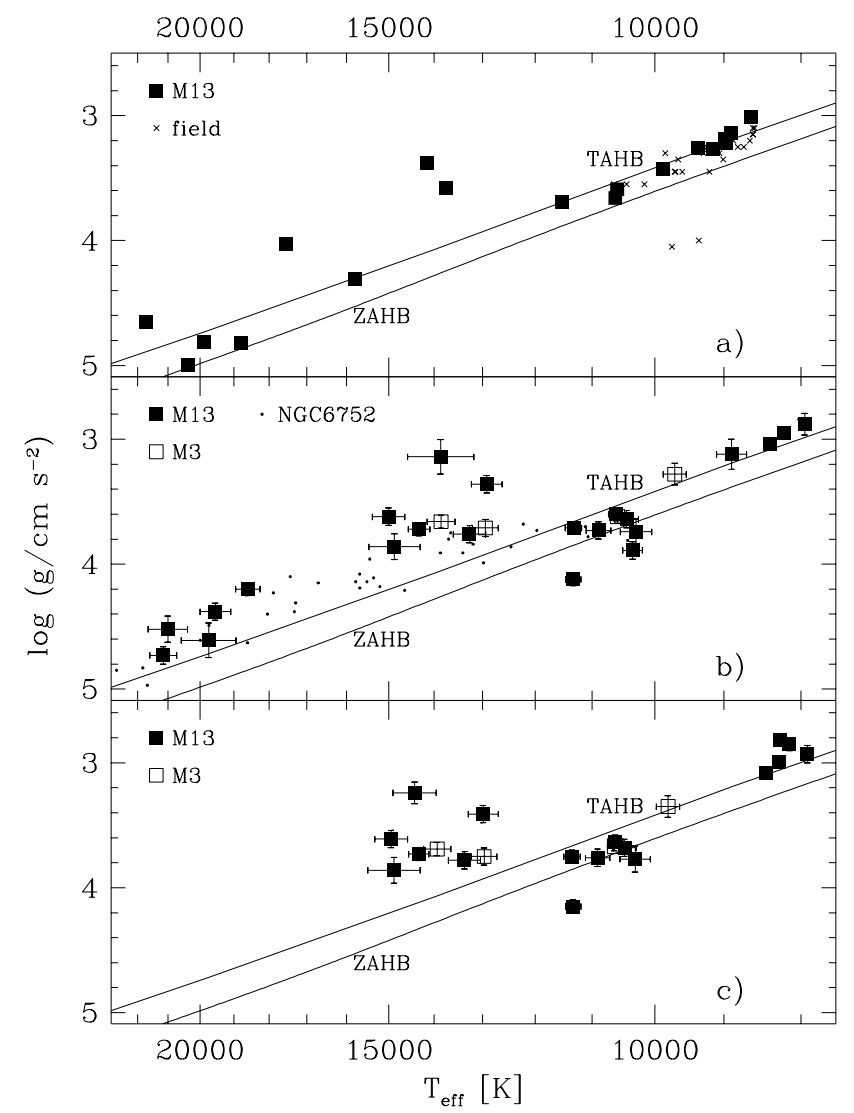

Fig. 3. Atmospheric parameters of the programme stars in $M 3$ and M 13 obtained with $\alpha$-enhanced metal-poor model atmospheres $([\mathrm{Fe} / \mathrm{H}]=-1.5,[\alpha / \mathrm{Fe}]+0.4)$. The solid lines mark the zero-age (ZAHB) and terminal-age (TAHB) loci of canonical HB tracks for $[\mathrm{M} / \mathrm{H}]=-1.54$. These loci define the region within which the HB models spend $99 \%$ of their HB lifetime. a) Temperatures and gravities from Strömgren $u v b y \beta$ photometry (for comparison we also show results for cool field BHB stars obtained with the same method by Kinman et al. 2000). b) Temperatures and gravities for all stars are derived from fits to the Balmer line profiles, using as starting values the photometrically determined parameters. The error bars note the formal $1 \sigma$ errors from the line profile fits. The model spectra used here do not include metal lines. For comparison we also show the results for blue tail stars in NGC 6752 from Moehler et al. (2000, obtained with metalpoor model spectra without metal lines). c) Temperatures and gravities of the programme stars cooler than $15000 \mathrm{~K}$ from Balmer line profile fits using model spectra that include metal lines for $[\mathrm{Fe} / \mathrm{H}]=-1.5$ and $[\alpha / \mathrm{Fe}]=+0.4$.

determined parameters also as starting values for the hotter stars, although the results of the line profile fitting depend very little on the starting values for temperatures above about $12000 \mathrm{~K}$.

For those stars that do not have Strömgren photometry we used $B-V$ from Tables 1 and 2 to estimate temperatures. As we only have $B-V$ we need additional information to decide on a combination of effective temperature and surface gravity. We therefore forced the parameters obtained from $B-V$ to agree with the canonical zero-age HB model from Sweigart (1997b) for $[\mathrm{M} / \mathrm{H}]=-1.5$. These parameters (listed in Table 3) should therefore be taken with many grains of salt and are useful mainly as starting points for the line profile fitting. They are therefore not plotted in the top panel of Fig. 3. The $B-V$ colour of B 352 in M 3 is very probably wrong, as it suggests an effective temperature of well above $25000 \mathrm{~K}$, in which case one would expect an absolute brightness of about +4.2 instead of +1 m. 9 .

\subsection{Line profile fitting}

We first computed model atmospheres using ATLAS9 (Kurucz 1993) for $[\mathrm{Fe} / \mathrm{H}]=-1.5$ and $[\alpha / \mathrm{Fe}]=+0.4$, using a mixing length parameter of 1.25 and no overshoot. This way our models should be identical to those of Castelli (1998) used for the analysis of the Strömgren photometry and also to those used by Kinman et al. (2000) for the analysis of field horizontal branch stars. Then we used Lemke's version ${ }^{1}$ of the LINFOR program (developed originally by Holweger, Steffen, and Steenbock at Kiel University) to compute a grid of theoretical spectra which include the Balmer lines $\mathrm{H}_{\alpha}$ to $\mathrm{H}_{22}$ and $\mathrm{He} \mathrm{I}$ lines, but no metal lines. The grid covered the range $7000 \mathrm{~K} \leq T_{\text {eff }} \leq 15000 \mathrm{~K}$, $2.5 \leq \log g \leq 5.5$ at a solar helium abundance $\log \frac{n_{\mathrm{He}}}{n_{\mathrm{H}}}=-1.0$. We also computed a grid with identical abundances, but no convection, for $7000 \mathrm{~K} \leq T_{\text {eff }} \leq 35000 \mathrm{~K}, 2.5 \leq \log g \leq 5.5$. This grid was used for all stars with photometric temperatures above $12000 \mathrm{~K}$, where convection should have vanished.

In Table 3 (Cols. 5 and 6) we list the results obtained from fitting the Balmer lines $\mathrm{H}_{\beta}$ to $\mathrm{H}_{10}$ (excluding $\mathrm{H}_{\epsilon}$ to avoid the Ca II $\mathrm{H}$ line) with these model spectra. To establish the best fit, we used the routines developed by Bergeron et al. (1992) and Saffer et al. (1994), as modified by Napiwotzki et al. (1999), which employ a $\chi^{2}$ test. The $\sigma$ necessary for the calculation of $\chi^{2}$ is estimated from the noise in the continuum regions of the spectra. The fit program normalizes model spectra and observed spectra using the same points for the continuum definition. The errors given in Tables 3 and 4 are rms errors derived from the $\chi^{2}$ fit (see Moehler et al. 1999 for more details). These errors are obtained under the assumption that the only error source is statistical noise (derived from the continuum of the spectrum). However, errors in the normalization of the spectrum, imperfections of flat field/sky background correction, variations in the resolution (e.g. due to seeing variations when using a rather large slit width) and other effects may produce systematic rather than statistical errors, which are not well represented by the error obtained from the fit routine. As the helium abundance is fixed in the model spectra we did not try to fit any helium lines, because this would introduce strong biases in case of non-solar helium abundances (expected for the hot HB stars, see Sect. 5). The results of this analysis are plotted in Fig. 3 (central panel). As it is not precisely clear at which temperature convection truly vanishes in HB stars we fitted stars with photometric temperatures between $9000 \mathrm{~K}$ and $12000 \mathrm{~K}$ with both the convective and the non-convective model spectra. The non-convective model spectra yield effective temperatures lower by on average $10 \mathrm{~K}$ and surface gravities lower by on average 0.015 dex. Thus the effect of convection at these temperatures is certainly negligible for our analysis.

\footnotetext{
${ }^{1}$ For a description see http://a400. sternwarte. uni-erlangen.de/ ai26/linfit/linfor.html
} 
Table 3. Atmospheric parameters for our programme stars in M 3 (B) and M 13 (G, WF) as derived from photometry (Cols. 2 and 3 ) and fits to the Balmer line profiles using metal-poor, $\alpha$-enhanced model atmospheres with solar helium abundances. Columns 5 and 6 list the results obtained with model spectra which contain only hydrogen and helium lines, while Cols. 7 and 8 give the results obtained with model spectra that also include metal lines for $[\mathrm{Fe} / \mathrm{H}]=-1.5$ and $[\alpha / \mathrm{Fe}]=+0.4$. For those stars where we could obtain a meaningful fit we also obtained iron abundances from spectrum synthesis (Cols. 4 and 9, see text for details). Given are abundances using microturbulent velocities of $3 \mathrm{~km} \mathrm{~s}^{-1}$ and (in brackets) for $0 \mathrm{~km} \mathrm{~s}^{-1}$. The errors are statistical errors only.

\begin{tabular}{|c|c|c|c|c|c|c|c|c|}
\hline \multirow[t]{2}{*}{ Star } & \multicolumn{2}{|c|}{ Photometry } & \multirow[t]{2}{*}{$\log \epsilon_{\mathrm{Fe}}$} & \multicolumn{4}{|c|}{ Line profiles } & \multirow[t]{2}{*}{$\log \epsilon_{\mathrm{Fe}}$} \\
\hline & $\begin{array}{l}T_{\text {eff }} \\
{[\mathrm{K}]}\end{array}$ & $\begin{array}{r}\log g \\
{\left[\mathrm{~cm} \mathrm{~s}^{-2}\right]}\end{array}$ & & $\begin{array}{l}T_{\text {eff }} \\
{[\mathrm{K}]}\end{array}$ & $\begin{array}{r}\log g \\
{\left[\mathrm{~cm} \mathrm{~s}^{-2}\right]}\end{array}$ & $\begin{array}{l}T_{\text {eff }} \\
{[\mathrm{K}]}\end{array}$ & $\begin{array}{r}\log g \\
{\left[\mathrm{~cm} \mathrm{~s}^{-2}\right]}\end{array}$ & \\
\hline G43 & 13050 & 2.60 & & $13900 \pm 710$ & $3.14 \pm 0.14$ & $14400 \pm 450$ & $3.24 \pm 0.09$ & - \\
\hline G129 & 8630 & 3.01 & $5.7(5.6)$ & $8000 \pm 70$ & $2.88 \pm 0.09$ & $7900 \pm 50$ & $2.93 \pm 0.07$ & $5.1(5.1)$ \\
\hline G137 & 8910 & 3.14 & $6.4(6.8)$ & $8400 \pm 50$ & $3.04 \pm 0.02$ & $8400 \pm 50$ & $3.08 \pm 0.02$ & $6.0(6.4)$ \\
\hline G195 & 8980 & 3.19 & $5.8(5.8)$ & $8200 \pm 40$ & $2.95 \pm 0.03$ & $8300 \pm 40$ & $2.99 \pm 0.02$ & $5.2(5.2)$ \\
\hline G201 & 9360 & 3.26 & $6.4(6.6)$ & $8900 \pm 200$ & $3.12 \pm 0.12$ & $8300 \pm 50$ & $2.82 \pm 0.03$ & $5.5(5.6)$ \\
\hline G208 & 9870 & 3.43 & $6.6(6.6)$ & $10900 \pm 200$ & $3.73 \pm 0.07$ & $10900 \pm 200$ & $3.76 \pm 0.07$ & $6.9(7.0)$ \\
\hline G219 & 8970 & 3.22 & $5.8(6.0)$ & $10300 \pm 160$ & $3.89 \pm 0.07$ & $8100 \pm 60$ & $2.85 \pm 0.05$ & $4.9(5.2)$ \\
\hline G235 & 9150 & 3.27 & $5.9(5.8)$ & $11300 \pm 130$ & $4.12 \pm 0.05$ & $11300 \pm 130$ & $4.15 \pm 0.05$ & $6.8(6.7)$ \\
\hline G297 & 10590 & 3.59 & & $10600 \pm 130$ & $3.60 \pm 0.05$ & $10600 \pm 120$ & $3.63 \pm 0.05$ & $6.5(6.6)$ \\
\hline G314 & 10620 & 3.66 & & $10400 \pm 190$ & $3.64 \pm 0.07$ & $10500 \pm 180$ & $3.68 \pm 0.07$ & $6.0(6.0)$ \\
\hline G322 & 13750 & 3.58 & & $15000 \pm 370$ & $3.62 \pm 0.07$ & $14900 \pm 360$ & $3.61 \pm 0.07$ & $7.4(7.5)$ \\
\hline G342 & 11520 & 3.69 & & $11300 \pm 140$ & $3.71 \pm 0.05$ & $11300 \pm 140$ & $3.75 \pm 0.05$ & $6.2(6.1)$ \\
\hline G382 & 14160 & 3.38 & & $14300 \pm 240$ & $3.72 \pm 0.05$ & $14300 \pm 240$ & $3.73 \pm 0.05$ & $8.0(8.0)$ \\
\hline G496 & 17560 & 4.03 & & $18600 \pm 350$ & $4.20 \pm 0.05$ & & & - \\
\hline G503 & 15800 & 4.31 & & $14900 \pm 580$ & $3.86 \pm 0.10$ & $14900 \pm 570$ & $3.86 \pm 0.10$ & $7.4(7.6)$ \\
\hline G635 & 18800 & 4.82 & & $19500 \pm 450$ & $4.38 \pm 0.07$ & & & - \\
\hline G744 & 19880 & 4.81 & & $21000 \pm 620$ & $4.52 \pm 0.10$ & & & - \\
\hline G781 & 20390 & 5.00 & & $19700 \pm 820$ & $4.61 \pm 0.14$ & & & - \\
\hline G827 & 21720 & 4.65 & & $21200 \pm 430$ & $4.73 \pm 0.07$ & & & - \\
\hline WF 3035 & 9550 & 3.51 & & $10300 \pm 240$ & $3.74 \pm 0.10$ & $10300 \pm 240$ & $3.77 \pm 0.10$ & - \\
\hline WF 3085 & 10260 & 3.66 & & $12900 \pm 300$ & $3.36 \pm 0.07$ & $12900 \pm 300$ & $3.41 \pm 0.07$ & $7.5(8.2)$ \\
\hline WF 3485 & 9790 & 3.57 & & $13300 \pm 330$ & $3.76 \pm 0.07$ & $13400 \pm 330$ & $3.78 \pm 0.07$ & $7.9(8.4)$ \\
\hline B254 & 9630 & 3.53 & & $10600 \pm 110$ & $3.62 \pm 0.05$ & $10600 \pm 110$ & $3.67 \pm 0.03$ & $6.3(6.2)$ \\
\hline B352 & 28600 & 5.63 & & $13900 \pm 300$ & $3.66 \pm 0.05$ & $13900 \pm 300$ & $3.69 \pm 0.05$ & $8.1(8.6)$ \\
\hline B445 & 9850 & 3.58 & & $9700 \pm 170$ & $3.28 \pm 0.09$ & $9800 \pm 170$ & $3.35 \pm 0.09$ & $6.2(6.2)$ \\
\hline B621 & 10170 & 3.64 & & $12900 \pm 260$ & $3.71 \pm 0.07$ & $13000 \pm 250$ & $3.75 \pm 0.07$ & $7.6(7.9)$ \\
\hline
\end{tabular}

\subsubsection{Stars hotter than $12000 \mathrm{~K}$}

The star G43 is found to have a high temperature $\left(T_{\mathrm{eff}} \approx\right.$ $14000 \mathrm{~K})$ which, along with its bright visual magnitude $(y=14$. 26), suggests that it is a UV-bright star. The star is clearly seen as such on the $1600 \AA$ A image of M 13 obtained with the Ultraviolet Imaging Telescope (UIT, Parise et al. 1998), but the crowding prevents a good determination of its ultraviolet magnitude. Using the $y$ magnitude and the derived effective temperature, and assuming an apparent distance modulus of $(m-M)_{V}=14.51$ toward M 13 and a reddening of $E_{B-V}=0.02$, we estimate a luminosity of $\log L / L_{\odot}=2.47$. The evolutionary status of G43 is uncertain, but given the large extreme HB (EHB) population in M 13, it may be either a He-shell burning post-EHB star, or a post-early AGB star (cf. Moehler et al. 1998). In any case, since G43 is almost certainly not an HB star, it is excluded from further discussion in this paper.

The results from the Balmer line profile analyses place the stars hotter than $12000 \mathrm{~K}$ further away from the ZAHB than the results from the Strömgren colours (see Fig. 3, central panel). These stars, however, show also weaker He I lines than predicted by model atmospheres with solar helium abundance, while in the cooler stars the He I lines - where detectable - are well described by line profiles for solar helium abundance. The offset from the canonical tracks seen in Fig. 3 for the stars hotter than about $12000 \mathrm{~K}$ in both M 3 and M 13 is already well known from other globular clusters (see, e.g. Moehler 2001). To better illustrate this general behavior, we also give the results for the blue HB stars in NGC 6752 obtained with similar methods by Moehler et al. (2000). Note that the gravities of the blue HB stars in M 3, M 13 and NGC 6752 all follow the same trend with $T_{\text {eff. }}$ Possible reasons for this behaviour are discussed in Sects. 5 and 6.

\subsubsection{Stars cooler than $12000 \mathrm{~K}$}

As can be seen from the top and central panel in Fig. 3 and from Table 3, there is an excellent agreement between the values of $T_{\text {eff }}$ and $\log g$ derived from Strömgren photometry, and those derived from a detailed Balmer line fit for the three stars (G297, G314, G342) in M 13 with Strömgren temperatures between $10000 \mathrm{~K}$ and $12000 \mathrm{~K}$. This agreement provides evidence in support of the Strömgren calibration of Castelli (1998) for deriving the atmospheric parameters of low-metallicity hot HB stars in this temperature range. The atmospheric 
parameters of these stars as well as B254 also agree well with canonical zero-age HB (ZAHB).

As can be seen in the top panel of Fig. 3, the stars with Strömgren temperatures cooler than $\approx 10000 \mathrm{~K}$ show a small offset towards low gravities from the canonical HB. Figure 1 shows that at least the two coolest stars might actually be somewhat evolved as they are brighter than the main $\mathrm{HB}$ in that region of colour-magnitude diagram. There is also good agreement with gravities derived for field blue HB stars in this temperature range by Kinman et al. (2000).

Comparing the top and central panel of Fig. 3, however, shows that there are significant discrepancies between the results obtained from photometric and from spectroscopic data for these stars (cf. Table 3). The results from the Balmer line profile fitting move the cool BHB stars to hotter and cooler temperatures, thereby producing a gap between about $9000 \mathrm{~K}$ to $10000 \mathrm{~K}$.

While these stars are not the central target of our investigation we followed the suggestions of the referee to look for causes that might explain this behaviour. Readers interested in the hot stars in M 3 and M 13 may skip the remainder of this section and move directly to Sect. 5.

The Balmer line profile fitting routine could not find a a model spectrum that provides a good fit to all Balmer lines in two cases (G235 and WF3035, both below the ZAHB in Fig. 3): Models at about $10000 \mathrm{~K}$ to $11000 \mathrm{~K}$ predict too weak $\mathrm{H}_{\delta}$ to $\mathrm{H}_{\beta}$ lines, whereas models at about $8000 \mathrm{~K}$ predict too weak $\mathrm{H}_{10}$ to $\mathrm{H}_{8}$ lines. The spectra of all other cool stars are very well reproduced by the model fits. We checked the following possible causes for problems in our Balmer line fitting:

Synthetic Spectra: We compared "our" temperature-pressure distribution and resulting theoretical Balmer profiles for selected models with those computed by Castelli (available on http: //kurucz.harvard.edu/) and those computed using the program SYNSPEC (Hubeny \& Lanz 2003) and found no discernible differences.

Metal lines: The Balmer line fitting procedure we used was originally developed for hot white dwarfs, which show only hydrogen and/or helium lines. Blue HB stars below $10000 \mathrm{~K}$, however, may show strong metal lines even in case of metal-poor stars, which may affect the shape of the Balmer lines especially when observed at low resolution. To rule out the influence of metal lines on the line profile fitting for the cooler stars we produced a grid of model spectra with $[\mathrm{Fe} / \mathrm{H}]=-1.5$ and $[\alpha / \mathrm{Fe}]=+0.4$ (with and without convection for the same temperature ranges as above), which include metal lines (up to effective temperatures of $15000 \mathrm{~K}$ ) and which we used for stars with photometric temperature estimates below $15000 \mathrm{~K}$. The results of fits with these model atmospheres are plotted in the bottom panel of Fig. 3 and listed in Table 3 (Cols. 7 and 8). Two stars show large changes (G201 moves from $8900 \mathrm{~K}$ to $8300 \mathrm{~K}, \mathrm{G} 219$ moves from $10300 \mathrm{~K}$ to $8100 \mathrm{~K}$ ) and the $\chi^{2}$ of all stars is significantly reduced. However, the discrepancies with the evolutionary tracks persist as well as the bad fits for G 235 and WF 3035 .
Signal-to-Noise and/or resolution: Our data have $S / N$ between 40 and 60 (determined from the continuum between $4500 \AA$ and $4600 \AA$ ) and a resolution of $3.4 \AA$. We convolved a theoretical spectrum from our grid with metal lines to this resolution and added noise to achieve a $S / N$ of 40 and 60 , respectively. Fitting this spectrum reproduced the original parameters $(9000 \mathrm{~K}, \log g=3.0)$ to within $\pm 100 \mathrm{~K}$ in $T_{\text {eff }}$ and \pm 0.02 dex in $\log g$.

Assuming that the real resolution might be better than determined from the wavelength calibration frames (which illuminate the whole slit of 1.'5) we assumed a resolution of $3.0 \AA$ for another test, both with the theoretical model spectrum as with our data. Again the effect is small, with changes of less than $50 \mathrm{~K}$ in $T_{\text {eff }}$ and 0.02 dex in $\log g$. We also verified that the shape of the wavelength calibration lines is well described by a Gaussian.

Rotation: Assuming a projected rotational velocity of $20 \mathrm{~km} \mathrm{~s}^{-1}$ does not affect the results, which is not unexpected considering the limited resolution of our spectra.

So we currently have no explanation for the disagreement between the atmospheric parameters for the cool stars derived from Strömgren photometry, and those derived from Balmerline fitting.

\section{Radiative levitation of heavy elements}

As described in Moehler et al. (2000) and Behr et al. (1999, 2000), hot HB stars show helium depletion and iron enrichment for effective temperatures above $11000 \mathrm{~K}$ to $12000 \mathrm{~K}$. To verify this behaviour from our low-resolution spectra, we performed a spectrum synthesis to reproduce the iron lines in the wavelength region 4490-4590 A (as described in Moehler et al. 2000). First estimates are obtained using the atmospheric parameters given in Cols. 7 and 8 of Table 3 and are listed in Col. 9 of the same table. The best fitting theoretical spectra are shown in Fig. 4 together with the observed spectra. While it is obvious from Fig. 4 that the cool stars show significant iron lines our fits (using the atmospheric parameters from the Balmer line profile fits) indicate very low iron abundances. If we use the atmospheric parameters derived from Strömgren photometry instead the iron abundances are much closer to $[\mathrm{Fe} / \mathrm{H}] \approx-1.5$ (see Col. 4 of Table 3 ). We take this as further evidence that the results from the Balmer line profile fits cannot be trusted for stars cooler than about $9500 \mathrm{~K}$ and we will omit these stars (including WF 3035 due to its fit problems) from all further discussion, as they are not the central goal of this paper.

In order to be at least roughly consistent with the actual stellar abundances, we redetermined $T_{\text {eff }}, \log g$ and $\log \frac{n_{\mathrm{He}}}{n_{\mathrm{H}}}$ for the stars showing evidence for enriched iron abundances using model atmospheres with super-solar metallicity $([\mathrm{M} / \mathrm{H}]=+0.5)$, varying helium abundance and no convection. This was done by fitting the Balmer lines $\mathrm{H}_{\beta}$ to $\mathrm{H}_{10}$ (excluding $\mathrm{H}_{\epsilon}$ to avoid the $\mathrm{Ca}$ II $\mathrm{H}$ line) and the He I lines $4026 \AA$, $4388 \AA$, $4471 \AA$, and $4921 \AA$ with these metal-rich model atmospheres. The results are given in Cols. 2-4 of Table 4. Using these new 
Table 4. Atmospheric parameters for our programme stars in M 3 (B) and M 13 (G, WF) as derived from fits to Balmer and helium lines using enriched scaled-solar model spectra $([\mathrm{M} / \mathrm{H}]=+0.5)$. Columns $2-4$ give the results obtained with model spectra containing only hydrogen and helium lines, Cols. 6-8 give the results obtained with model spectra including metal lines. For those stars where we could obtain a meaningful fit we also obtained iron abundances from spectrum synthesis (Col. 5, see text for details). Given are abundances using microturbulent velocities of $3 \mathrm{~km} \mathrm{~s}^{-1}$ and (in brackets) for $0 \mathrm{~km} \mathrm{~s}^{-1}$. The errors are statistical errors only.

\begin{tabular}{|c|c|c|c|c|c|c|c|}
\hline \multirow[t]{2}{*}{ Star } & \multicolumn{3}{|c|}{ Line profiles w/o metal lines } & \multirow[t]{2}{*}{$\log \epsilon_{\mathrm{Fe}}$} & \multicolumn{3}{|c|}{ Line profiles w metal lines } \\
\hline & $\begin{array}{c}T_{\text {eff }} \\
{[\mathrm{K}]}\end{array}$ & $\begin{array}{r}\log g \\
{\left[\mathrm{~cm} \mathrm{~s}^{-2}\right]}\end{array}$ & $\log \frac{n_{\mathrm{He}}}{n_{\mathrm{H}}}$ & & $\begin{array}{l}T_{\text {eff }} \\
{[\mathrm{K}]}\end{array}$ & $\begin{array}{r}\log g \\
{\left[\mathrm{~cm} \mathrm{~s}^{-2}\right]}\end{array}$ & $\log \frac{n_{\mathrm{He}}}{n_{\mathrm{H}}}$ \\
\hline G322 & $14600 \pm 400$ & $3.82 \pm 0.09$ & $-2.32 \pm 0.23$ & $7.4(7.5)$ & $14500 \pm 400$ & $3.84 \pm 0.09$ & $-2.41 \pm 0.28$ \\
\hline G382 & $14100 \pm 200$ & $3.94 \pm 0.05$ & $-2.07 \pm 0.12$ & $7.8(8.2)$ & $14100 \pm 300$ & $4.00 \pm 0.07$ & $-2.13 \pm 0.14$ \\
\hline G496 & $17700 \pm 400$ & $4.33 \pm 0.07$ & $-1.90 \pm 0.09$ & - & $17300 \pm 400$ & $4.35 \pm 0.07$ & $-1.92 \pm 0.09$ \\
\hline G503 & $15200 \pm 600$ & $4.20 \pm 0.12$ & $-2.60 \pm 0.42$ & $7.4(7.6)$ & $15100 \pm 500$ & $4.22 \pm 0.12$ & $-2.72 \pm 0.48$ \\
\hline G635 & $19200 \pm 600$ & $4.58 \pm 0.09$ & $-2.15 \pm 0.10$ & - & $18700 \pm 600$ & $4.59 \pm 0.09$ & $-2.16 \pm 0.12$ \\
\hline G744 & $20200 \pm 1100$ & $4.61 \pm 0.12$ & $-2.04 \pm 0.12$ & - & $18400 \pm 700$ & $4.57 \pm 0.12$ & $-1.94 \pm 0.12$ \\
\hline G781 & $18800 \pm 1000$ & $4.71 \pm 0.16$ & $-1.46 \pm 0.16$ & - & $17800 \pm 800$ & $4.70 \pm 0.16$ & $-1.37 \pm 0.17$ \\
\hline G827 & $20800 \pm 700$ & $4.89 \pm 0.09$ & $-2.37 \pm 0.10$ & - & $19800 \pm 600$ & $4.89 \pm 0.09$ & $-2.28 \pm 0.12$ \\
\hline WF 3085 & $13100 \pm 300$ & $3.65 \pm 0.09$ & $-2.34 \pm 0.35$ & $7.4(7.9)$ & $12800 \pm 300$ & $3.62 \pm 0.09$ & $-2.36 \pm 0.38$ \\
\hline WF 3485 & $13300 \pm 300$ & $4.05 \pm 0.09$ & $-2.31 \pm 0.38$ & $7.7(8.2)$ & $13300 \pm 300$ & $4.08 \pm 0.09$ & $-2.36 \pm 0.45$ \\
\hline B352 & $13800 \pm 300$ & $3.92 \pm 0.07$ & $-2.21 \pm 0.23$ & $8.1(8.5)$ & $13800 \pm 300$ & $3.99 \pm 0.07$ & $-2.29 \pm 0.29$ \\
\hline B621 & $13000 \pm 300$ & $3.98 \pm 0.07$ & $-2.20 \pm 0.31$ & $7.4(7.7)$ & $12800 \pm 300$ & $3.98 \pm 0.09$ & $-2.34 \pm 0.36$ \\
\hline
\end{tabular}

atmospheric parameters, we then repeated the iron abundance analysis and used those fits for Fig. 5.

We also determined magnesium abundances where possible from the equivalent width of the Mg II line at $4482 \AA$ (see Table 5).

The final iron abundances derived from our spectra are listed in Col. 9 of Table 3 for stars cooler than $12000 \mathrm{~K}$ and in Col. 5 of Table 4 for stars hotter than $12000 \mathrm{~K}$ and are plotted in the central panel of Fig. 5. For consistency we always plot the abundances obtained with a microturbulent velocity of $3 \mathrm{~km} \mathrm{~s}^{-1}$, although $0 \mathrm{~km} \mathrm{~s}^{-1}$ would be more appropriate for the stars affected by diffusion. A drastic change in the iron abundance between $11500 \mathrm{~K}$ and $13000 \mathrm{~K}$ is obvious, in good agreement with the findings of Glaspey et al. (1989) for two hot HB stars in NGC 6752 and Behr et al. (1999, 2000) for hot HB stars in M 13 and M 15. The results in Fig. 5 show that all stars hotter than $12000 \mathrm{~K}$ for which we can estimate the iron abundance show evidence for radiative levitation. The iron abundance for the hotter stars is a factor of 50-100 greater than that of the cluster in general and consistent with that required to explain the Stromgren $u$-jump discussed by Grundahl et al. $\left(1999, \log \epsilon_{\mathrm{Fe}}=8.1\right)$. We also find a one-to-one correspondence between the position of a star relative to the $u$-jump (blueward/redward) and its iron abundance (enhanced/cluster abundance) whenever we were able to determine an iron abundance (cf. Fig. 6).

The upper panel of Fig. 5 shows that the onset of radiative levitation is accompanied by a drop in the helium abundance by $1 \mathrm{dex}$ or more. As the He I lines are very weak at about $10000 \mathrm{~K}$ we did not try to determine helium abundances for the cool stars. We note, however, that the observed He I lines in these cool stars agree well with those predicted for solar helium abundance and thus with the results of
Table 5. Magnesium abundances for our programme stars in M 3 (B) and M $13(\mathrm{G}, \mathrm{WF})$ for microturbulent velocities of $3 \mathrm{~km} \mathrm{~s}^{-1}$ and (in brackets) $0 \mathrm{~km} \mathrm{~s}^{-1}$. We also give the atmospheric parameters used for the abundance determinations.

\begin{tabular}{lrrrr}
\hline \hline Star & $\begin{array}{r}T_{\text {eff }} \\
{[\mathrm{K}]}\end{array}$ & $\begin{array}{r}\log g \\
{\left[\mathrm{~cm} \mathrm{~s}^{-2}\right]}\end{array}$ & $\log \frac{n_{\mathrm{He}}}{n_{\mathrm{H}}}$ & $\begin{array}{r}\log \epsilon_{\mathrm{Mg}} \\
3 \mathrm{~km} \mathrm{~s}^{-1}\left(0 \mathrm{~km} \mathrm{~s}^{-1}\right)\end{array}$ \\
\hline G297 & 10600 & 3.63 & -1.00 & $6.2(6.5)$ \\
G314 & 10400 & 3.68 & -1.00 & $6.0(6.2)$ \\
G322 & 14600 & 3.82 & -2.32 & $6.2(6.3)$ \\
G342 & 11300 & 3.75 & -1.00 & $5.9(6.2)$ \\
G382 & 14100 & 3.94 & -2.07 & $6.1(6.3)$ \\
G496 & 17700 & 4.33 & -1.90 & $6.2(6.3)$ \\
G503 & 15200 & 4.20 & -2.60 & $6.7(6.9)$ \\
G635 & 19200 & 4.58 & -2.15 & $6.9(7.0)$ \\
WF 3085 & 13100 & 3.65 & -2.34 & $6.3(6.6)$ \\
WF 3485 & 13300 & 4.05 & -2.31 & $6.0(6.1)$ \\
\hline B254 & 10600 & 3.67 & -1.00 & $6.4(6.7)$ \\
B352 & 13800 & 3.92 & -2.21 & $6.1(6.2)$ \\
B445 & 9800 & 3.35 & -1.00 & $6.0(6.3)$ \\
B621 & 13000 & 3.98 & -2.20 & $6.2(6.4)$ \\
\hline
\end{tabular}

Behr et al. (1999, 2000) from high resolution spectroscopy. Clearly the stable stellar atmosphere required for radiative levitation also permits the gravitational settling of helium.

The mean magnesium abundance for the stars cooler than $12000 \mathrm{~K}$ is $[\mathrm{Mg} / \mathrm{H}]=-1.4$ and for the hotter stars it is -1.2 . So the magnesium abundance does not change significantly during the onset of diffusion. The same result was also found by Moehler et al. (2000) for NGC 6752 and Behr et al. (1999, 2000) for M 13 and M 15. 


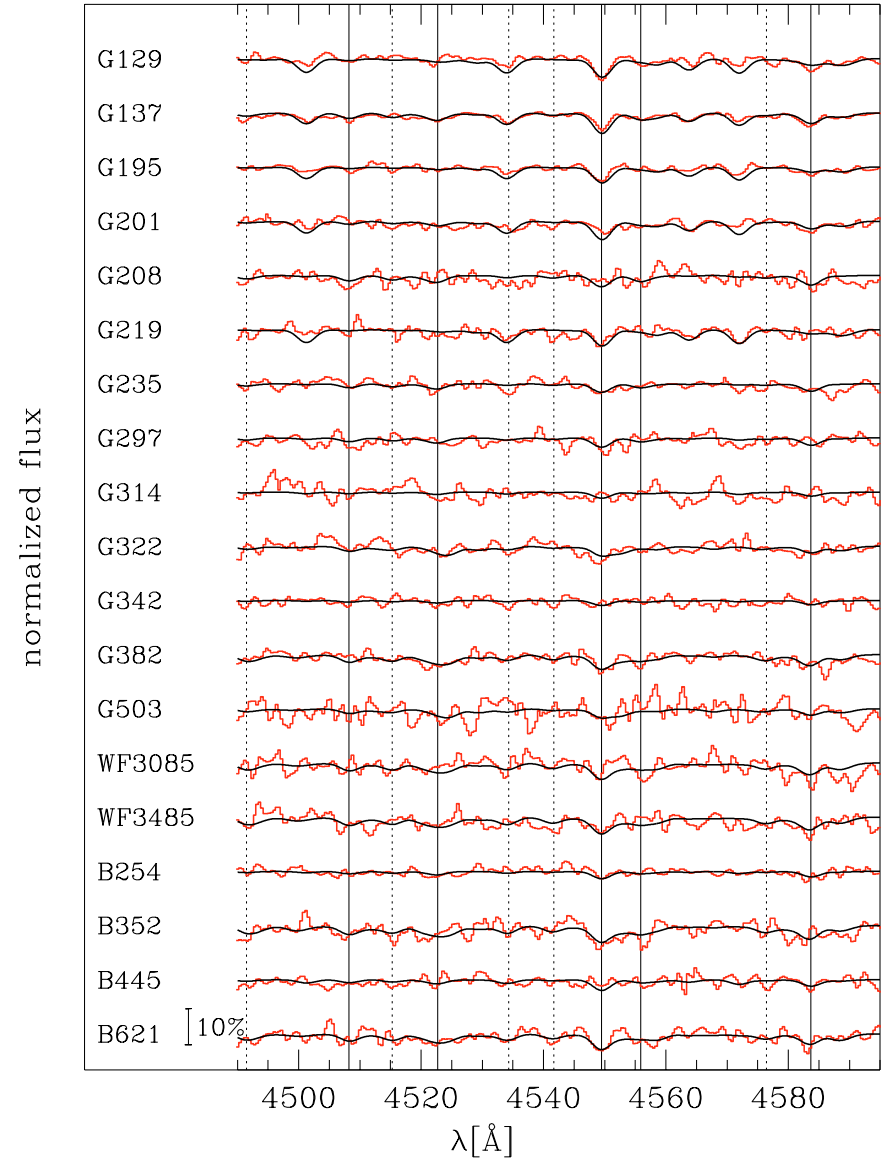

Fig. 4. Normalized spectra of the programme stars in M 13 and M 3 and theoretical spectra for the atmospheric parameters (from the Balmer line profiles) and iron abundances listed in Table 3. For stars hotter than $12000 \mathrm{~K}$ we show the fits obtained for atmospheric parameters from metal-rich model atmosphere fits. The solid and dotted lines mark the strongest and moderately strong iron lines in the spectra.

Rey et al. (2001) have suggested that radiative levitation and helium diffusion may be responsible for the formation of a blue tail in M 13. However, we find that the HB stars in M 3 show the same abundance pattern with respect to effective temperature as the stars in M 13 and NGC 6752. The fact that M 3 contains stars affected by radiative levitation but does not have a blue tail provides strong evidence against the Rey et al. (2001) suggestion.

The stellar parameters derived for the stars hotter than $12000 \mathrm{~K}$ from the metal-rich model atmospheres are plotted in the upper panel of Fig. 7 and compared to the canonical HB locus for a helium abundance $Y$ of 0.23 and a scaledsolar metallicity $[\mathrm{M} / \mathrm{H}]$ of -1.54 . As already noted by Moehler et al. (2000), using scaled-solar metal-rich model atmospheres moves the stars hotter than $12000 \mathrm{~K}$ closer to the canonical ZAHB, substantially reducing the low gravity offset seen in Fig. 3. However, it is obvious that discrepancies still exist between atmospheric parameters derived from observations and the predictions of canonical HB theory.

The model spectra calculated from the metal-rich model atmospheres and used for the results in the upper panel of Fig. 7 did not include metal lines. In order to determine if the

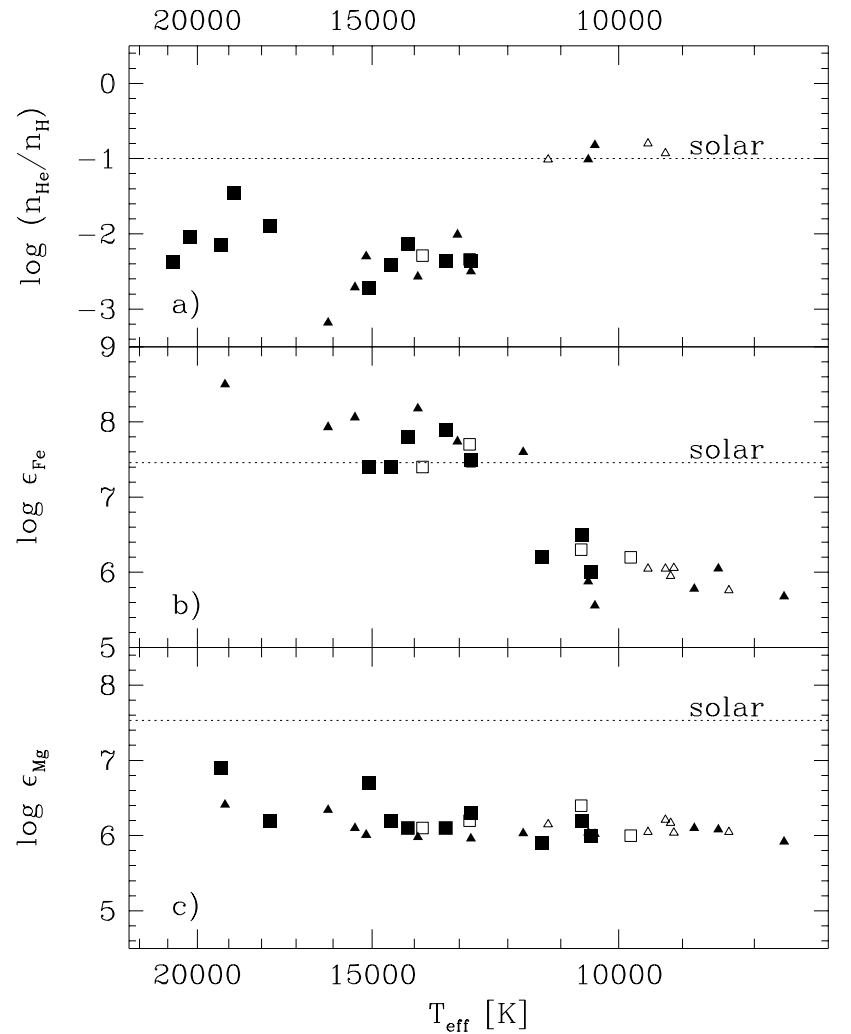

Fig. 5. Abundances of a) helium, b) iron, and c) magnesium for the programme stars in M 3 (open squares) and M 13 (filled squares). Also given are the results of Behr (priv. comm.) for stars in M 3 (open triangles) and M 13 (filled triangles).

strong metal lines that show up at the onset of diffusion might influence our results, we calculated a new grid of metal-rich model spectra, which include metal lines, for temperatures up to $23000 \mathrm{~K}$, and then used these spectra to fit all stars with effective temperatures above $12000 \mathrm{~K}$. The results are plotted in Fig. 7 (lower panel) and listed in Table 4 (Cols. 6-8). While the differences for stars cooler than $16000 \mathrm{~K}$ are small, the hotter stars become considerably cooler when fitted with theoretical spectra that include metal lines. This shift in temperature yields consistency of the hottest stars with canonical HB evolution tracks. One should remember, however, that these spectra were computed using scaled-solar abundances. It is very unlikely that diffusion will produce an overall enrichment of elements - highly non-solar abundance ratios are much more probable. So these results, while promising, should be taken with a grain of salt.

Also, as can be seen from Fig. 9, the masses of the stars obtained from their values of $T_{\text {eff }}$ and $\log g$ in Tables 3 (Cols. 7 and 8 ) and 4 (Cols. 6 and 7) tend to be too low. Comparing the derived masses to those expected for a star on the ZAHB at the same temperature yields a mean mass ratio of $0.9 \pm 0.2$ for the 5 stars below $12000 \mathrm{~K}$ (analysed with metal-poor model spectra including metal lines) and $0.8 \pm 0.2$ for the 12 hotter stars when analysed with metal-rich model spectra including metal lines. We used distance moduli of $(m-M)_{V}=14$ m.51 (M 13) and 15.02 (M 3) to derive these masses. 


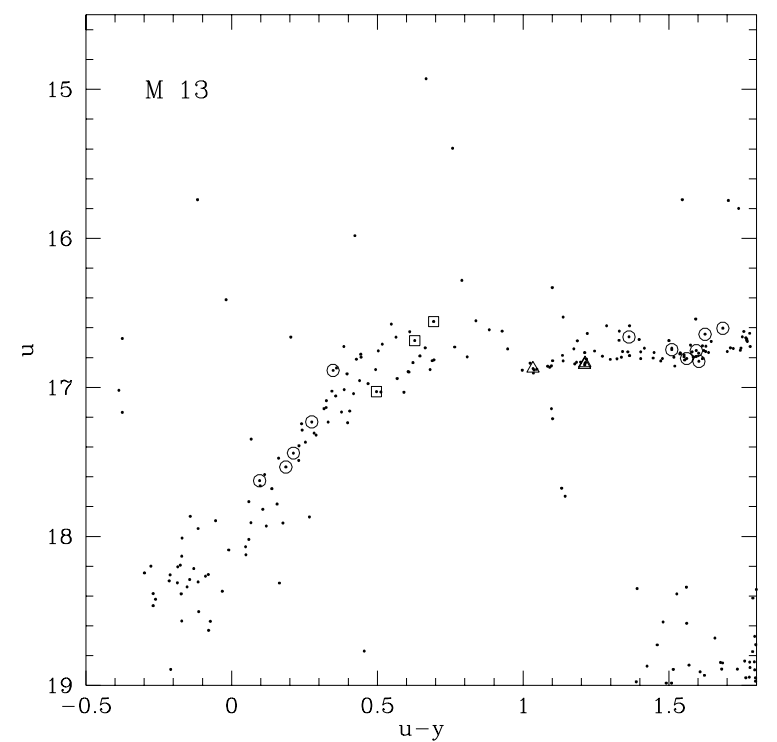

Fig. 6. The $u$-jump in M 13 with the spectroscopic targets marked: Open triangles mark stars with low iron abundance $([\mathrm{Fe} / \mathrm{H}] \approx-1.5)$, open squares mark stars with high iron abundance $([\mathrm{Fe} / \mathrm{H}] \approx+0.5)$. Stars for which the iron abundance could not be determined due to the limited $S / N$ (hot stars) or uncertainties in the atmospheric parameters (cool stars) are marked by open circles.

We conclude that both the gravities and masses of the HB stars between $12000 \mathrm{~K}$ and $16000 \mathrm{~K}$ remain about 0.15 0.2 dex too low compared to canonical predictions even when the stars are analyzed with metal-rich model spectra which include metal lines. This remaining offset may reflect a systematic error caused by our use of metal-rich model atmospheres with scaled-solar abundances. The results of Behr et al. $(1999,2000)$ have demonstrated that radiative levitation can lead to severely nonsolar abundance ratios in blue HB stars. Quite possibly, the use of scaled-solar metal-rich model atmospheres may not sufficiently approximate the actual atmospheric abundances in these stars. In addition, at such a high $([\mathrm{Fe} / \mathrm{H}]=+0.5)$ metal abundance, the model atmospheres are not well tested, and are more sensitive to inadequacies in the opacity distribution function than are models at lower abundances. Also a stratification of the stellar atmosphere influences the line profiles and our model atmospheres assume homogeneous atmospheres. Another possibility, suggested by Vink \& Cassisi (2002), is that an enhanced stellar wind in the radiatively levitated HB stars may alter the wings of the Balmer line profiles, leading to an underestimate of the surface gravity. While it remains to be seen if radiative levitation can be effective at the high mass loss rates obtained by Vink \& Cassisi (2002), their results do raise potential concerns about the use of hydrostatic model atmospheres.

\section{Effects of varying helium abundance}

As mentioned in Sect. 1, M 13 shows strong abundance variations along its red giant branch, which might be attributed to either deep mixing extending into the hydrogen-burning shell (helium mixing) or pollution of the stars with the ejecta from an earlier generation of AGB stars (helium pollution).

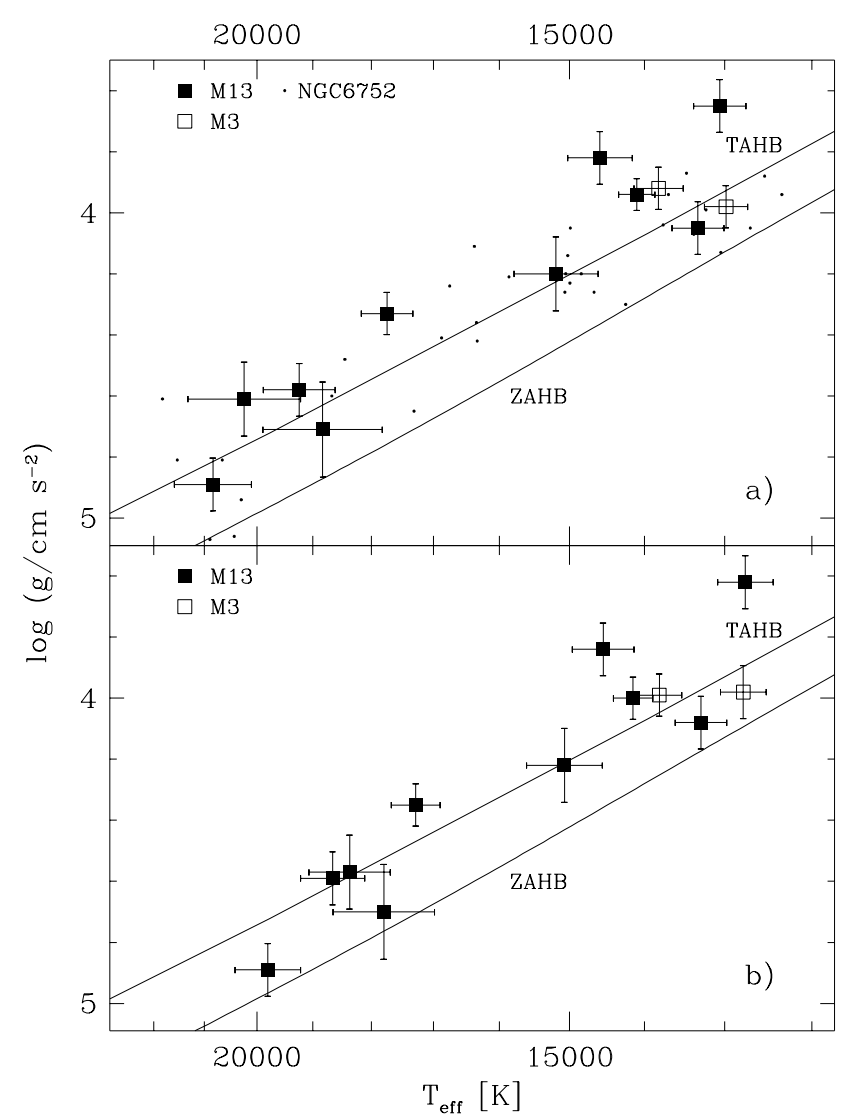

Fig. 7. Temperatures and gravities of the programme stars that show evidence for iron enrichment. The solid lines mark the zero-age (ZAHB) and terminal-age (TAHB) loci of canonical HB tracks for $[\mathrm{M} / \mathrm{H}]=-1.54$. These loci define the region within which the HB models spend $99 \%$ of their HB lifetime. a) Here we adopted a super-solar metallicity $([\mathrm{M} / \mathrm{H}]=+0.5)$ for the model atmospheres (see Sect. 5 for details), but did not include metal lines in the theoretical spectra. For comparison we also show the results for blue tail stars in NGC 6752 from Moehler et al. (2000, obtained with metal-rich model spectra without metal lines). b) These are the results obtained with metal-rich model spectra that include metal lines.

Both processes would increase the envelope helium abundance of the RGB stars with important consequences for the subsequent $\mathrm{HB}$ evolution. Here we discuss the potential impact of these effects on the properties of the blue HB stars in M 13.

\subsection{Helium mixing}

To illustrate the effects of helium mixing, we will use a set of mixed sequences computed by Moehler et al. (2000) for a main-sequence helium abundance $Y$ of 0.23 and a scaled-solar metallicity $[\mathrm{M} / \mathrm{H}]$ of -1.54 . These sequences were evolved up the RGB for different amounts of helium mixing using the approach of Sweigart (1997a). Mass loss was included according to the Reimers formulation with the mass-loss parameter $\eta_{\mathrm{R}}$ set equal to 0.45 . The evolution was then followed through the helium flash to the end of the HB phase using standard techniques. The HB locus of these helium-mixed sequences in the $\log g-T_{\text {eff }}$ plane is shown in the top panel of Fig. 8. 


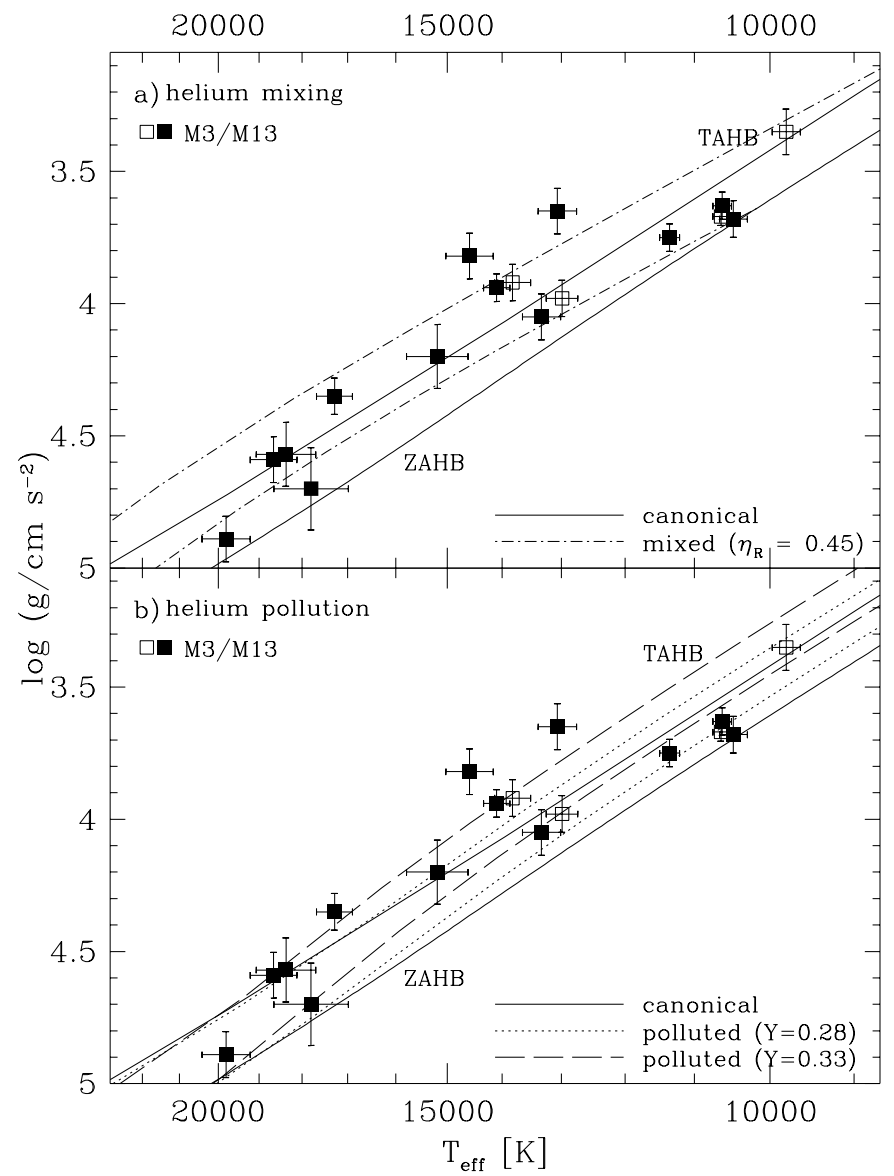

Fig. 8. Temperatures and gravities of the programme stars in M 3 and M 13 (from metal-rich model atmospheres for stars hotter than $12000 \mathrm{~K}$ and metal-poor model atmospheres for cooler stars, in both cases including metal lines) compared to non-canonical evolutionary models. The zero-age and terminal-age $\mathrm{HB}$ predicted by canonical models for $Y=0.23$ and $[\mathrm{M} / \mathrm{H}]=-1.54$ are plotted as solid lines. a) The zero-age and terminal-age $\mathrm{HB}$ for $[\mathrm{M} / \mathrm{H}]=-1.54$ computed with helium mixing for a Reimers mass-loss parameter $\eta_{\mathrm{R}}=0.45$ (see Sect. 6.1 for details). b) The zero-age and terminal-age HB computed with helium pollution for helium abundances of $Y=0.28$ and $Y=0.33$ (see Sect. 6.2 for details).

Since mixing increases the RGB mass loss due to a brighter RGB tip luminosity, a mixed model will arrive on the HB at a higher effective temperature than the corresponding canonical model. At the same time mixing increases the envelope helium abundance in the HB model, which leads to a higher energy output of the hydrogen-burning shell and hence to a brighter surface luminosity (Sweigart \& Gross 1976).

The net effect is to shift the mixed locus in Fig. 8 towards lower gravities with increasing effective temperature relative to the canonical locus, until a maximum offset is reached for $15000 \mathrm{~K}<T_{\text {eff }}<20000 \mathrm{~K}$. At higher temperatures the mixed locus shifts back towards the canonical locus, as the luminosity of the hydrogen-burning shell declines due to the decreasing envelope mass. The predicted locus along the extreme HB (EHB) does not depend strongly on the extent of the mixing, since the luminosities and gravities of the EHB stars are primarily determined by the mass of the helium core, which is

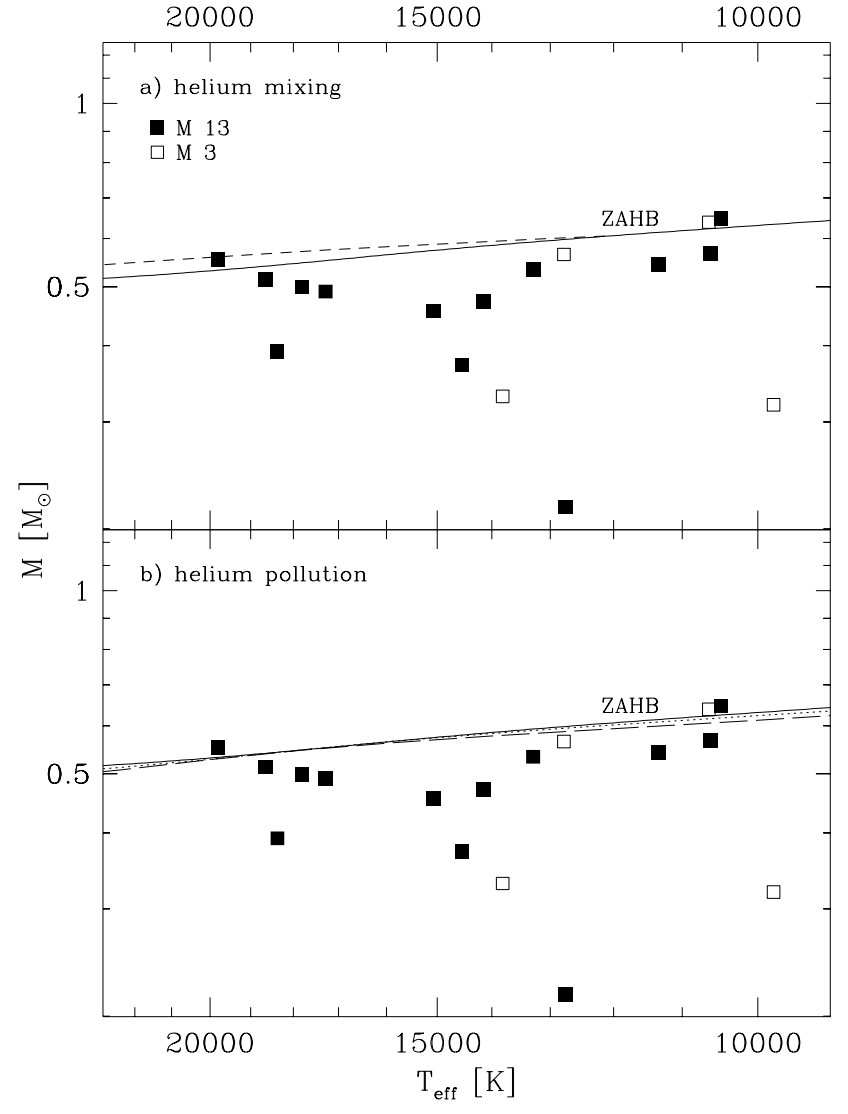

Fig. 9. Temperatures and masses of the programme stars in $M 3$ and M 13 (from metal-rich model atmospheres for stars hotter than $12000 \mathrm{~K}$ and metal-poor model atmospheres for cooler stars; in both cases metal lines were included in the theoretical spectra) compared to evolutionary tracks. a) The solid line marks the canonical ZAHB and the mixed ZAHB is given by the short-dashed line (see Sect. 6.1 for details). b) Again the solid line marks the canonical ZAHB and the polluted ZAHBs are given by the dotted $(Y=0.28)$ and long-dashed line $(Y=0.33)$, respectively (see Sect. 6.2 for details).

nearly the same for the mixed and canonical models. Models at the cool end of the mixed locus will also differ little from the canonical models, since the cool HB stars will have undergone little mixing on the RGB. This explains why the mixed and canonical loci converge at lower temperatures in Fig. 8. The size of the offset between the mixed and canonical loci depends on the assumed value of $\eta_{\mathrm{R}}$, becoming larger as $\eta_{\mathrm{R}}$ decreases (see Moehler et al. 2000).

\subsection{Helium pollution}

The ejecta of AGB stars which have undergone hot bottom burning should be enriched in helium (Ventura et al. 2001). Thus the pollution of either pre-stellar material or existing lowmass stars by this material will increase either the overall helium abundance or at least that in the envelopes. D' Antona et al. (2002) have studied the evolution of stars that form out of polluted material in which the helium abundance was enriched from a canonical value of $Y=0.23-0.24$ to $Y=0.29$. They found that while the effects on the pre-HB evolution are quite small, the HB morphology itself is strongly affected by helium 
pollution. The main effect is to shift the HB stars to hotter temperatures due to smaller turnoff masses for a given cluster age and to increased mass loss on the RGB. Polluted stars should thus be found primarily among the hottest HB stars. Indeed, D'Antona et al. (2002) suggest that helium pollution is responsible for the extended blue tails found in such clusters as M 13 and NGC 6752.

To investigate the effects of helium pollution, we computed two additional sets of $\mathrm{HB}$ sequences for $[\mathrm{M} / \mathrm{H}]=-1.54$ in which the helium abundance was increased by 0.05 and 0.10 above our canonical value of $Y=0.23$. The HB loci defined by these helium-polluted sequences are plotted in the bottom panel of Fig. 8. The gravity offset between these loci and the canonical locus is primarily due to the luminosity difference between the helium-polluted and canonical models.

HB stars derive their energy from two sources: helium burning in the core and hydrogen burning in a shell. Since an increase in the initial helium abundance leads to a decrease in the helium-core mass (Sweigart \& Gross 1978), the energy output of the helium core will be lower in a helium-polluted model than in a canonical model. Along the EHB, where hydrogenshell burning is unimportant, a helium-polluted star will therefore be fainter and have a higher gravity than a canonical star. The opposite is true at cooler temperatures where the hydrogenburning shell is a major energy source. The higher envelope helium abundance of a helium-polluted star then leads to a brighter surface luminosity and thus to a lower gravity. At the transition between the blue HB and the EHB $\left(T_{\text {eff }} \approx 20000 \mathrm{~K}\right)$, the gravities of the canonical and helium-polluted models are virtually identical. This is the point where the higher hydrogenburning luminosity of a helium-polluted star offsets the lower helium-burning luminosity.

One aspect of the helium-polluted loci in Fig. 8 requires further comment. According to the helium-pollution scenario outlined by D'Antona et al. (2002), the stars at the cool end of an M 13-like HB, i.e., those near the top of the blue tail, would not be helium polluted. Thus in an actual cluster one would expect the cooler HB stars to lie within the canonical locus. However, as one goes to higher temperatures along the blue tail, the fraction of the HB stars that are helium polluted would increase, and thus the locus predicted by the D'Antona et al. scenario would shift away from the canonical locus towards the lower gravities of the helium-polluted loci in Fig. 8. This shift to lower gravities is not seen in Fig. 8, because the heliumpolluted loci in this figure assume that all of the HB stars including the cooler stars are helium polluted.

\subsection{Comparison with observations}

In Figs. 8 and 9 we compare the effective temperatures, surface gravities and masses derived from metal-rich $\left(T_{\text {eff }}>12000 \mathrm{~K}\right)$ and metal-poor $\left(T_{\text {eff }}<12000 \mathrm{~K}\right)$ model atmospheres to the scenarios described above. While the helium-mixed models provide a better description of the temperatures and gravities derived for the stars between $12000 \mathrm{~K}$ and $16000 \mathrm{~K}$ than the canonical models, they do not resolve the problem with the low observed masses. The same is also true for the helium-polluted models, where we again find that the observed masses are systematically too low. The fact that neither the helium-mixed nor helium-polluted models can account for the low masses raises doubt about the reality of any gravity and/or temperature discrepancies between the programme stars in Fig. 8 and canonical predictions. Without a better understanding of possible systematic errors, it is not possible to argue for or against the above noncanonical scenarios, given the present data.

\section{Conclusions}

We have obtained low-resolution ( $3.4 \AA$ ) spectroscopy of 22 hot HB candidates in M 13, and four in M 3, in order to derive atmospheric parameters (effective temperatures and surface gravities) as well as abundances of helium, magnesium, and iron. One star (G43) in M 13 turned out to be a UVbright star, while the remaining targets appear to be bona-fide HB stars. For the stars between $10000 \mathrm{~K}$ and $12000 \mathrm{~K}$, the atmospheric parameters derived from fitting the Balmer lines and from Strömgren photometry are in good agreement. For the stars cooler than $10000 \mathrm{~K}$, there is a discrepancy between parameters derived from Strömgren photometry and those derived from fitting the Balmer lines. We suspect that there are problems in the Balmer line fitting because the derived temperatures show a gap between about $9000 \mathrm{~K}$ to $10000 \mathrm{~K}$, and because the derived iron abundances show a wide scatter. However, despite numerous tests, we were unable to determine the cause of the disagreement in the derived parameters.

For stars hotter than $12000 \mathrm{~K}$ in both clusters, we find evidence for helium depletion and a large iron enrichment, consistent with the results on M 13 by Behr et al. (1999) and on NGC 6752 by Moehler et al. (2000). The similar temperatures for the onset of radiative levitation in M 3, M 13, and NGC 6752 suggest that this phenomenon is unrelated to the HB morphology. Instead, the onset of radiative levitation may be connected to the disappearance of the surface convection zone, as suggested by Sweigart (2002).

We compare our temperature and gravity results with the predictions of the helium mixing scenario of Sweigart (1997b) and the helium pollution scenario of D'Antona et al. (2002). These scenarios are attractive because they can explain the origins of the HB blue tail in globular clusters such as M 13 without requiring a fine-tuning of the mass loss, and because they can relate the blue tail to the observed abundance anomalies on the RGB. Both scenarios predict lower gravities (and larger luminosities) for stars near $15000 \mathrm{~K}$. For stars cooler than $12000 \mathrm{~K}$, we find the observed gravities in agreement with canonical models. This result is consistent with the work of Caloi (2001), who compared the HB luminosities of M 3 and M 13 to conclude that there was no evidence for a substantial surface helium abundance increase for HB stars near the temperature of the RR Lyrae stars.

For stars hotter than the onset of radiative levitation at $12000 \mathrm{~K}$, we fit the Balmer lines using metal-rich $([\mathrm{Fe} / \mathrm{H}]=+0.5)$ model atmospheres to derive the temperature and gravity. Although the use of metal-rich atmospheres reduces the discrepancy with canonical models, we still find an offset to lower gravity of about 0.2 dex for stars between 
$12000 \mathrm{~K}$ and $16000 \mathrm{~K}$, similar to what was seen at temperatures hotter than $15000 \mathrm{~K}$ in NGC 6752 by Moehler et al. (2000). However, there are a couple of reasons why this result should be viewed with caution. First, the derived masses for these stars appear to be too low compared to either the canonical or the helium-enriched scenarios. Second, the metalenriched atmospheres used to derive these parameters are not well-tested, and do not take into account the strongly non-solar abundance ratios. Future high-resolution spectroscopy could help reduce these possible systematic errors in gravity, by allowing the atmospheric parameters and abundances to be determined iteratively, using model atmospheres with non-solar abundance ratios.

Acknowledgements. We want to thank the staff of the Calar Alto observatory for their support and B. Behr for making his abundance measurements available to us. We are very grateful to F. Castelli and M. Lemke for their help with the model atmospheres for the cool HB stars. Thanks go also to our referee R.C. Peterson, whose comments improved this paper considerably. This work was supported by the DLR under grant 50 OR 96029-ZA.

\section{References}

Behr, B. B., Cohen, J. G., McCarthy, J. K., \& Djorgovski, S. G. 1999, ApJ, 517, L135

Behr, B. B., Cohen, J. G., \& McCarthy, J. K. 2000, ApJ, 531, L37

Bergeron, P., Saffer, R. A., \& Liebert, J. 1992, ApJ, 394, 228

Buonanno, R., Corsi, C. E., Buzzoni, A., et al. 1994, A\&A, 290, 69

Caloi, V. 2001, A\&A, 366, 91

Cannon, R. D., Croke, B. F. W., Bell, R. A., Hesser, J. E., \& Stathakis, R. A. 1998, MNRAS, 298, 601

Castelli, F. 1998, MemSAI, 69, 165

Catelan, M., \& de Freitas, \& Pacheco, J. A. 1995, A\&A, 297, 345

Cavallo, R. M., \& Nagar, N. M. 2000, AJ, 120, 1364

D’Antona, F., Caloi, V., Montalbán, J., Ventura, P., \& Gratton, R. 2002, A\&A, 395, 69

Dolphin, A. 2000, PASP, 112, 1383

Ferraro, F. R., Paltrinieri, B., Fusi Pecci, F., et al. 1997, ApJ, 484, L145

Glaspey, J. W., Michaud, G., Moffat, A. F. J., \& Demers, S. 1989, ApJ, 339,926

Gratton, R. G., Bonifacio, P., Bragaglia, A., et al. 2001, A\&A, 369, 87

Grundahl, F. 1999, in Spectrophotometric Dating of Stars and Galaxies, ed. I. Hubeny, S. R. Heap, \& R. H. Cornett, ASP Conf. Ser., 192, 223

Grundahl, F., VandenBerg, D. A., \& Anderson, M. I. 1998, ApJ, 500, L179

Grundahl, F., Catelan, M., Landsman, W. B., Stetson, P. B., \& Andersen, M. 1999, ApJ, 524, 242
Hamuy, M., Walker, A. R., Suntzeff, N. B., et al. 1992, PASP, 104, 533

Harris, W. E. 1996, AJ, 112, 1487 (version, June, 22 1999)

Horne, K. 1986, PASP, 98, 609

Hubeny, I., \& Lanz, T. 2003, ApJS, in press (June 2003)

Kinman, T., Castelli, F., Cacciari, C., et al. 2000, A\&A, 364, 102

Kraft, R. P. 1999, Ap\&SS, 265, 153

Kraft, R. P., Sneden, C., Langer, G. E., \& Prosser, C. F. 1992, AJ, 104, 645

Kraft, R. P., Sneden, C., Langer, G. E., \& Shetrone, M. D. 1993, AJ, 106,1490

Kraft, R. P., Sneden, C., Langer, G. E., Shetrone, M. D., \& Bolte, M. 1995, AJ, 109, 2586

Kraft, R. P., Sneden, C., Smith, G. H., et al. 1997, AJ, 113, 279

Kurucz, R. L. 1993, ATLAS9 Stellar Atmospheres Program and $2 \mathrm{~km} \mathrm{~s}^{-1}$ grid, CD-ROM No. 13, http://kurucz.harvard.edu/

Lupton, R. H., Gunn, J. E., \& Griffin, R. F. 1987, AJ, 93, 1114

Michaud, G., Vauclair, G., \& Vauclair, S. 1983, ApJ, 267, 256

Moehler, S., Landsman, W. B., \& Napiwotzki, R. 1998, A\&A, 335, 510

Moehler, S., Sweigart, A. V., \& Catelan, M. 1999, A\&A, 351, 519

Moehler, S., Sweigart, A. V., Landsman, W. B., \& Heber, U. 2000, A\&A, 360, 120

Moehler, S. 2001, PASP, 113, 1162

Napiwotzki, R., Schönberner, \& Wenske, V. 1993, A\&A, 268, 653

Napiwotzki, R., Green, P. J., \& Saffer, R. A. 1999, ApJ, 517, 399

Oke, J. B. 1990, AJ, 99, 1621

Paltrinieri, B., Ferraro, F. R., Fusi Pecci, F., \& Carretta, E. 1998, MNRAS, 293, 434

Parise, R. A., Bohlin, R. C., Neff, S. G., et al. 1998, ApJ, 501, L67

Piotto, G., King, I. R., \& Djorgovski, S. G., et al. 2002, A\&A, 391, 945

Pryor, C. P., Latham, D. W., \& Hazen, M. L. 1988, AJ, 96, 123

Rey, S.-C., Yoon, S.-J., Lee, Y.-W., Chaboyer, B., \& Sarajedini, A. 2001, AJ, 122, 3219

Saffer, R. A., Bergeron, P., Koester, D., \& Liebert, J. 1994, ApJ, 432, 351

Sarajedini, A., Chaboyer, B., \& Demarque, P. 1997, PASP, 109, 1321

Sweigart, A. V. 1997a, ApJ, 474, L23

Sweigart, A. V. 1997b, in The Third Conference on Faint Blue Stars, ed. A. G. D. Philip, J. Liebert, \& R. A. Saffer (L. Davis Press: Schenectady), 3

Sweigart, A. V. 2002, in JD 5, XXIV IAU GA, Highlights of Astronomy, 12, in press [astro-ph/0103133]

Sweigart, A. V., \& Gross, P. G. 1976, ApJS, 32, 367

Sweigart, A. V., \& Gross, P. G. 1978, ApJS, 36, 405

Tüg, H. 1977, ESOMe, 11, 7

VandenBerg, D. A. 2000, ApJS, 129, 315

Ventura, P., D’Antona, F., Mazzitelli, I., \& Gratton, R. 2001, ApJ, 550, L65

Vink, J. S., \& Cassisi, S. 2002, A\&A, 392, 553 